IMA Journal of Applied Mathematics (2012) Page 1 of 41

doi:10.1093/imamat/hxs057

\title{
Networks of Self-Adaptive Dynamical Systems
}

\author{
JULIO RODRIGUEZ*
}

Faculté des Sciences et Techniques de l'Ingénieur (STI), Institut de Microtechnique (IMT), Laboratoire de Production Microtechnique (LPM), Ecole Polytechnique Fédérale de Lausanne (EPFL), CH-1015

Lausanne, Switzerland and Fakultät für Physik, Universität Bielefeld, D-33501 Bielefeld, Germany

*Corresponding author. Email: julio.rodriguez@epfl.ch and jrodrigu@physik.uni-bielefeld.de

AND

MAX-Olivier HONGLER

Faculté des Sciences et Techniques de l'Ingénieur (STI), Institut de Microtechnique (IMT), Laboratoire de Production Microtechnique (LPM), Ecole Polytechnique Fédérale de Lausanne (EPFL),

CH-1015 Lausanne, Switzerland

Email: max.hongler@epfl.ch

[Received on 14 January 2011; revised on 20 June 2012; accepted on 23 July 2012]

We discuss the adaptive behaviour of a collection of heterogeneous dynamical systems interacting via a weighted network. At each vertex, the network is endowed with a dynamical system with individual (initially different) control parameters governing the local dynamics. We then implement a class of network interactions which generates a self-adaptive behaviour, driving all local dynamics to adopt a set of consensual values for their local parameters. While for ordinary synchronization each individual dynamical system is restored to its original dynamics once network interactions are removed, here the consensual values of control parameters are definitively acquired-even if interactions are removed. For a wide class of dynamical systems, we show analytically how such a plastic and self-adaptive training of control parameters can be realized. We base our study on local dynamics characterized by dissipative ortho-gradient vector fields encompassing a vast class of attractors (in particular limit cycles). The forces generated by the coupling network are derived from a generalized potential. A set of numerical experiments enables us to observe the transient dynamics and corroborate the analytical results obtained.

Keywords: ortho-gradient dynamics; mixed canonical-dissipative systems; limit cycle oscillators; selfadaptive mechanisms; networks' adjacency and Laplacian matrices; Lyapunov method.

'Tu dépasses sans te perdre

Les frontières de ton corps'

Paul Eluard

\section{Introduction}

Bio-mechanical systems (e.g. the leg or arm of a robot) are often modelled by damped vibrating systems

$$
\begin{aligned}
& \dot{x}_{v}=-\bar{\lambda} y_{v}-\mathrm{f} x_{v}+x_{o}(t), \\
& \dot{y}_{v}=-\bar{\lambda} x_{v}-\mathrm{f} y_{v}+y_{o}(t),
\end{aligned}
$$


where $x_{o}(t)$ and $y_{o}(t)$ are the output signals of the locomotion controller given by a limit cycle oscillator, one of the simplest forms being

$$
\begin{aligned}
& \dot{x}_{o}=-\lambda y_{o}-\left(x_{o}^{2}+y_{o}^{2}-1\right) x_{o}, \\
& \dot{y}_{o}=-\lambda x_{o}-\left(x_{o}^{2}+y_{o}^{2}-1\right) y_{o} .
\end{aligned}
$$

The limit cycle oscillator (i.e. locomotion controller) excites the damped vibrating system in order to produce locomotion. To ensure the maximum amplitude response of $x_{v}(t)$ (e.g. the maximum leg stride), the locomotion controller's frequency must be set at the vibrating systems' resonant frequency (here $\bar{\lambda}=\lambda$ ). However, due to changing characteristics of the bio-mechanical system (e.g. mass changesadded load, length changes - growth), detuning occurs and the locomotion controller will no longer excite the vibrating system at the resonant frequency. Hence, as suggested in Buchli \& Ijspeert (2004) and Righetti et al. (2006), the locomotion controller must automatically adapt $\bar{\lambda}$ to ensure resonancethat is, $\lambda$ itself becomes time-dependent (i.e. $\lambda \rightsquigarrow \lambda(t)$ ) and has its own dynamics. Building on the example presented in Righetti et al. (2006), we propose an adaptive frequency oscillator as

$$
\begin{aligned}
& \dot{x}_{o}=-\lambda y_{o}-\left(x_{o}^{2}+y_{o}^{2}-1\right) x_{o}, \\
& \dot{y}_{o}=-\lambda x_{o}-\left(x_{o}^{2}+y_{o}^{2}-1\right) y_{o}, \\
& \dot{\lambda}=-\left(y_{v} x_{o}-x_{v} y_{o}\right),
\end{aligned}
$$

for which we have $\lim _{t \rightarrow \infty} \lambda(t)=\bar{\lambda}$, as numerous numerical investigations do indeed confirm.

Based on this motivation, here we implement adaptive mechanisms in a network of $N \geqslant 2$ coupled individual dynamical systems where all individual systems are allowed to self-adapt to each other. We first start by considering an $N$-vertex network where each vertex is equipped with an individual dynamical system. The $N$ local systems are additively coupled via time- and state-variable dependent edges. The global dynamical system reads

$$
\begin{aligned}
& \dot{X}_{k}=\underbrace{\mathrm{L}_{k}\left(X_{k} ; \Lambda_{k}\right)}_{\text {local dynamics }}+\underbrace{\mathrm{C}_{k}(X, \Delta)}_{\text {coupling dynamics }} k=1, \ldots, N, \\
& \dot{\Delta}=\underbrace{\mathrm{B}(X, \Delta)}_{\text {binding dynamics }}
\end{aligned}
$$

where $X_{k} \in \mathbb{R}^{p_{k}}$ are the state variables of the $k$ th node, $\mathrm{L}_{k}$ is a local vector field governing the local dynamics and $\Lambda_{k} \in \mathbb{R}^{q_{k}}$ are local control parameters. ${ }^{1}$ The coupling dynamics $\mathrm{C}_{k}(X ; \Delta) \in \mathbb{R}^{p_{k}}$ describe how the local dynamics interact. The notation is $X:=\left(X_{1}, \ldots, X_{N}\right)$, and $\Delta$ stands for the variables influencing the environment (e.g. coupling weights, edges between vertices of the network, etc.). The set $\Delta$ evolves according to the binding dynamics B. Note that Equations (1.1) is encompassed in the general framework exposed in Blekhman (1988).

Even for the simpler case involving functionally homogeneous dynamics $\mathrm{L}_{k} \equiv \mathrm{L}$ but heterogeneous control parameters $\Lambda_{k} \neq \Lambda_{j}$ for $j \neq k$, it is generally unknown when synchronized collective dynamical states may emerge from Equations (1.1). This question still motivates a wealth of papers, a field recently revived by the seminal contribution of Pecora \& Carroll (1998) who explicitly studied the network, induced synchronization capability. For diffusively coupled homogeneous dynamics $L_{k} \equiv L$ with

\footnotetext{
${ }^{1}$ We use the following notation: we use; to separate the variables from the parameters in the arguments of a function.
} 
$\Lambda_{k}=\Lambda_{j}$, the master stability equation proposed in Pecora \& Carroll (1998) allows to investigate the local stability of synchronized orbits characterized by $X_{k}(t)=X_{c}(t)$ for all $k$. For slight heterogeneity (i.e. small $\Lambda_{k}$ mismatches), the master stability equation still allows one to approximately characterize the emergence of synchronized motion - as corroborated by numerical probing in Hramov et al. (2008).

A common feature of all papers describing synchronization of systems with $\mathrm{L}_{k} \equiv \mathrm{L}$ but different $\Lambda_{k}$ is that the synchronized dynamical state is maintained by the permanent action of the network connections. Hence, if network interactions are switched off, synchronization is destroyed and each local system is restored to its individual and distinct evolution. In our present approach, we study an alternative class of dynamics for which the network interactions confer persistent features in the local dynamics, i.e. interactions induce 'plastic' deformations. As a result, if after a given time the interactions are removed, the local dynamics does not recover its original behaviour, but exhibits a permanent alteration. In our present models, this is realized via a self-adaptive learning rule which allows the $\Lambda_{k}$ parameters to evolve (i.e. adapt with time). The study of adaptation phenomena is an established research axis with wide interdisciplinary relevance. Among the wealth of available papers, the seminal work of Ermentrout (1991) and Acebrón \& Spigler's (1998) article are closely related to our present interest. Recently, Taylor et al. (2010) considered all-to-all coupled phase oscillators with an adaptive frequency mechanism. Our present models generalize (Taylor et al., 2010) with respect to both the local dynamics and the coupling networks (the all-to-all assumption is relaxed).

Technically, the implementation of a learning rule effectively enlarges the dimensionality of the global system by introducing additional parametric dynamics in Equations (1.1). This drives us to consider dynamical systems of the form

$$
\begin{aligned}
\dot{X}_{k} & =\underbrace{\mathrm{L}_{k}\left(X_{k}, \Lambda_{k}\right)}_{\text {local dynamics }}+\underbrace{\mathrm{C}_{k}(X, \Delta)}_{\text {coupling dynamics }} \\
\dot{\Delta} & =\underbrace{\mathrm{B}(X, \Delta)}_{\text {binding dynamics }} \quad k=1, \ldots, N, \\
\dot{\Lambda}_{k} & =\underbrace{\mathrm{P}_{k}(X, \Delta)}_{\text {parametric dynamics }}
\end{aligned}
$$

We thus confer to $\Lambda_{k}$ the status of new variables which we call parametric variables from now on. Note that by suitably renaming the variables, Equations (1.2) can be encompassed by Equations (1.1). Nevertheless, we insist on presenting the network dynamics in the form of Equations (1.2) as this explicitly isolates the self-adaptive mechanism that, via the network environment, plastically deforms the local parameters.

As initiated in Rodriguez \& Hongler (2009b) and in the case of homogeneous dynamics $\mathrm{L}_{k} \equiv \mathrm{L}$ (i.e. $\left|\Lambda_{k}\right|=q \forall k$ ), we focus on $\mathrm{P}_{k}$-dynamics that ultimately drive the $\Lambda_{k}$ towards a set of common and constant consensual valued parameters $\Lambda_{\mathrm{c}}$

$$
\lim _{t \rightarrow \infty} \Lambda_{k}(t)=\Lambda_{\mathrm{c}} \quad k=1, \ldots, N .
$$

By allowing local systems to ultimately acquire identical parameters, we are able to discuss analytically both the existence and the stability of the resulting 'ordered' consensual states. Apart from the analytical tractability of existence and stability, adapting local parameters is likely to enhance network synchronization. To this aim, adaptive mechanisms have been recently considered in De Lellis et al. (2010), Liu et al. (2010) and De Lellis et al. (2008). These authors focus on adaptive coupling 
dynamics alone (i.e. thus assuming $\mathrm{P}_{k} \equiv 0$ ) which therefore offers an alternative possibility. As far as network topology is considered, synchronization enhancement can, for example, be achieved either via a discrete optimization problem as in Liu et al. (2009) or by considering simulated annealing-based algorithms with performance measures such as the eigenratio of the Laplacian ('entangled' networks as in Donetti et al. (2005)) or an order parameter that considers the simulated output of a dynamical network as in Gorochowski et al. (2010).

Dynamical systems belonging to Equations (1.2) have been studied in Rodriguez \& Hongler (2009a) with Hopf oscillators as local dynamics and commutative evolution as their binding dynamics (cf. Boccaletti et al., 2006). Building on our introductory example, the paradigmatic illustration of the class of dynamics to be discussed is

$$
\begin{aligned}
& \dot{x}_{k}=\lambda_{k} y_{k}-\left(x_{k}^{2}+y_{k}^{2}-1\right) x_{k}-\sum_{j=1}^{N} l_{k, j} x_{j} \\
& \dot{y}_{k}=\underbrace{-\lambda_{k} x_{k}-\left(x_{k}^{2}+y_{k}^{2}-1\right) y_{k}}_{\text {local dynamics }}-\underbrace{\sum_{j=1}^{N} l_{k, j} y_{j}}_{\text {coupling dynamics }} k=1, \ldots, N, \\
& \dot{\lambda}_{k}=\underbrace{-\sum_{j=1}^{N} l_{k, j}\left(x_{j} y_{k}-y_{j} x_{k}\right)}_{\text {parametric dynamics }}
\end{aligned}
$$

where $l_{k, j}$ are the entries of the Laplacian matrix associated with the underlying network. For Equations (1.4), the set $\Lambda_{k}$ reduces to a single parameter $\lambda_{k}$ controlling the frequency of the $k$ th oscillator. The resulting dynamics 'plastically' modifies the values $\lambda_{k}$ and induces convergence towards a common and constant value $\lambda_{c}$ while, simultaneously, the coupling dynamics synchronizes the entire network. For our class of models, the convergence will be established by explicitly constructing ad hoc Lyapunov functions. The consensual frequency $\lambda_{c}$ is analytically calculated. It will be observed that for our dynamics $\lambda_{\mathrm{c}}$ only depends on the initial values (i.e. $\lambda_{k}(0)$ ), and not on the topology of the network. The network connectivity does, however, strongly control the convergence rate (i.e. the adaptive velocity).

Inspired by Equations (1.4), we introduce first in Section 2.1, ortho-gradient (O-G) local vector fields. This enables us to generate attractors that generalize the simple limit cycles. A non-Liouvillian dissipative mechanism is obtained via the gradient of a generalized local potential. This dissipative part is itself orthogonal to a 'driving' part which, when solenoidal, generates a canonical evolution. The distinction between a gradient and Hamiltonian vector field is discussed in Hirsch et al. (2004, Chapter 9). Our local systems include both dynamics simultaneously. The well-known mixed-canonical systems introduced in Hongler \& Ryter (1978), Schweitzer et al. (2001) and Schweitzer (2003) arising when the solenoidal part is a symplectic form is an example of our class. In this case $p$ is even to allow canonical conjugated variables. Suitable choices of the local potential (i.e. dissipative mechanisms) drive the solutions towards orbits solving the orthogonal part of the dynamics. Limit cycles and generally more complex attracting manifolds are asymptotically reached. The O-G system ensures that once on an attractor, the dissipative character of the dynamics vanishes. Note that the generalized Hamiltonian dynamical systems discussed in Nambu (1973) offer an alternative way to classify our dynamics by following the lines recently exposed in Frank (2010). Examples of O-G systems are given in Section 2.2. 
In Section 2.3, we introduce interactions between local systems by coupling dynamics that can also be derived from the gradient of a connection potential. Obviously, this potential intimately depends on the entries of an adjacency matrix characterizing the network connectivity. In Section 2.4, we distinguish between two types of control parameters according to their propensity to adapt. For parameters with a high propensity to adapt, we construct an adaptive mechanism (i.e. parametric dynamics) that enables us to analytically determine the ultimate consensual parameter state. In Section 3, we present sufficient conditions for the adaptive mechanism to drive the global system towards a consensual state. All stages of our constructions are illustrated by a collection of examples. Finally, in Section 5, numerical simulations illustrate the theoretical assertions. In addition, numerical experiments show the generic behaviour of our self-adaptive mechanisms when some of the specific hypothesis of our dynamics are relaxed.

\section{Networks of $\mathrm{O}-\mathrm{G}$ systems with parametric adaptivity}

In its general form, our global dynamical system is

$$
\begin{aligned}
& \dot{X}_{k}=\underbrace{\mathrm{D}_{k}\left(X_{k}, \Lambda_{k}\right)-\nabla \mathrm{E}_{k}\left(X_{k}\right)}_{\text {local dynamics }}-\underbrace{\mathrm{c}_{k} \frac{\partial \mathrm{V}}{\partial X_{k}}(X)}_{\text {coupling dynamics }} k=1, \ldots, N, \\
& \dot{\Lambda}_{k}=\underbrace{\mathrm{P}_{k}(X)}_{\text {parametric dynamics }} .
\end{aligned}
$$

(1) Local dynamics: local systems are of dimension $p$ and belong to the class of O-G systems (refer to Section 2.1).

(2) Coupling dynamics: the gradient of a given positive function characterizes the interactions (refer to Section 2.3).

(3) Parametric dynamics: through adaptive mechanisms, parameters are influenced by state variable interactions (refer to Section 2.4).

We now detail the constituent parts that compose our global dynamics.

\subsection{Local dynamics: $\mathrm{L}_{k}$}

All local dynamics belong to the class of O-G systems. This class of dynamical systems is split into two $p$-dimensional vector fields $\mathrm{D}_{k}$ and $\nabla \mathrm{E}_{k}$ and are defined as

$$
\mathrm{L}_{k}\left(X_{k} ; \Lambda_{k}\right):=\underbrace{\mathrm{D}_{k}\left(X_{k} ; \Lambda_{k}\right)}_{\text {orthogonal evolution }}-\underbrace{\nabla \mathrm{E}_{k}\left(X_{k}\right)}_{\text {gradient evolution }} k=1, \ldots, N,
$$

where $X_{k} \in \mathbb{R}^{p}$ are the state variables and $\Lambda_{k}$ is, for the time being, a set of $q_{k}$ fixed and constant parameters. Dissipation is due to the gradient evolution derived from a potential $\nabla \mathrm{E}_{k}$, the gradient of a potential $\mathrm{E}_{k}\left(X_{k}\right):=\frac{1}{2} \sum_{j \in I_{k}} \mathrm{G}_{j}\left(X_{k}\right)^{2}$ and $I_{k} \subseteq\{0, \ldots, p-1\}$. The real-valued functions $\mathrm{G}_{j}$ are sufficiently continuously differentiable and are defined such that the set

$$
\mathcal{L}_{k}:=\left\{X \in \mathbb{R}^{p} \mid \mathrm{G}_{j}(X)=0 j \in I_{k}\right\}
$$


is an $m_{k}$-dimensional compact submanifold ${ }^{2}$ with $m_{k}:=p-\left|I_{k}\right|$ ( $p$ minus the cardinality of the set of indices $I_{k}$ ).

On the orthogonal evolution $\mathrm{D}_{k}$, we impose the following orthogonality condition

$$
\left\langle\mathrm{D}_{k}\left(X_{k} ; \Lambda_{k}\right) \mid \nabla \mathrm{E}_{k}\left(X_{k}\right)\right\rangle=0 \quad \forall X_{k} \text { and } \forall \Lambda_{k} \text {. }
$$

This implies that one can arbitrarily fix the values for $\Lambda_{k}$ without destroying the orthogonality between $\mathrm{D}_{k}$ and $\nabla \mathrm{E}_{k}$. This orthogonality condition leads us to distinguish between two types of parameters.

(1) Parameters determining the time evolution on $\mathcal{L}_{k}$ are called flow parameters. They are explicitly written as $\Lambda_{k}$. They do not affect the geometric shape of $\mathcal{L}_{k}$ (i.e. they effectively define the time scale of the orbits on $\mathcal{L}_{k}$ ). They are characterized by the fact that they do not affect the orthogonality between the vector fields $\mathrm{D}_{k}$ and $\nabla \mathrm{E}_{k}$. Any O-G system has always at least one flow parameter (i.e. $\Lambda_{k}$ is not empty) since $D_{k}$ can always be multiplied by a scalar that fixes a time scale of the dynamics.

(2) Parameters determining the geometry of $\mathcal{L}_{k}$ are called geometric parameters. They simultaneously affect the potential $\mathrm{E}_{k}$ and $\mathrm{D}_{k}$, since the orthogonality of $\mathrm{D}_{k}$ with $\nabla \mathrm{E}_{k}$ must be maintained. In this contribution, geometric parameters will be held fixed and hence they are not explicitly written.

The following lemma characterizes the stability of the local dynamics. For simplicity's sake, we omit the index $k$ for the rest of this section.

Lemma 2.1 There exists a set $\mathcal{U} \supset \mathcal{L}$ such that all orbits solving

$$
\dot{X}=\mathrm{L}(X ; \Lambda)=\mathrm{D}(X ; \Lambda)-\nabla \mathrm{E}(X)
$$

with initial conditions in $\mathcal{U}$ converge towards $\mathcal{L}$.

Proof. In this proof, we will not explicitly write the parameters $\Lambda$. Since $\mathcal{L}$ is a submanifold, for all $X^{*} \in \mathcal{L}$, there exists a neighbourhood $\mathcal{U}_{X^{*}}$ of $X^{*}$. Let $\mathcal{U}$ be the union of all these neighbourhoods (i.e. $\left.\mathcal{U}=\bigcup_{X^{*} \in \mathcal{L}} \mathcal{U}_{X^{*}}\right)$. The convergence towards $\mathcal{L}$ follows from Lyapunov's second method with Lyapunov function

$$
\mathrm{E}(X)=\frac{1}{2} \sum_{j \in I} \mathrm{G}_{j}(X)^{2} .
$$

By construction, we have $\mathcal{L}=\left\{X \in \mathbb{R}^{p} \mid \mathrm{E}(X)=0\right\}$. Computing the time derivative

$$
\begin{aligned}
\langle\nabla \mathrm{E}(X) \mid \dot{X}\rangle & =\langle\nabla \mathrm{E}(X) \mid \mathrm{D}(X)-\nabla \mathrm{E}(X)\rangle \\
& =\underbrace{\langle\nabla \mathrm{E}(X) \mid \mathrm{D}(X)\rangle}_{=0}-\|\nabla \mathrm{E}(X)\|^{2} \leqslant 0 .
\end{aligned}
$$

\footnotetext{
${ }^{2}$ By definition, $\mathcal{L}_{k}$ is not empty, and for all $X^{*} \in \mathcal{L}_{k}$ there exists a neighbourhood $\mathcal{U}_{X^{*}} \subset \mathbb{R}^{p}$ of $X^{*}$ such that for all $X \in \mathcal{U}_{X}{ }^{*}$ the $\left(p-m_{k}\right) \times p$ Jacobian $\mathfrak{D G}_{k}(X)$ has rank $p-m_{k}\left(\mathfrak{D}\right.$ stands for the derivative operator and $\vec{G}_{k} \equiv\left(\mathrm{G}_{j_{1}}, \ldots, \mathrm{G}_{j_{p-m_{k}}}\right)$ with $j_{s} \in I_{k}$, $s=1, \ldots, p-m_{k}$ and $\left.p-m_{k}=\left|I_{k}\right|\right)$. Since $\mathcal{L}_{k}$ is the preimage of the closed set $\mathbf{0} \in \mathbb{R}^{p-m_{k}}$ by a continuous function $\overrightarrow{\mathrm{G}}_{k}$, it is closed. We assume it is bounded (there exists $b>0$ such that $\|X\| \leqslant b$ for all $X \in \mathcal{L}_{k}$ ), and thus $\mathcal{L}_{k}$ is compact.
} 
We then have

$$
\|\nabla \mathrm{E}(X)\|^{2}=0 \Longleftrightarrow \sum_{s=1}^{p}\left(\sum_{j \in I} \mathrm{G}_{j}(X) \frac{\partial \mathrm{G}_{j}}{\partial x_{s}}(X)\right)^{2}=0 \Longleftrightarrow \sum_{j \in I} \mathrm{G}_{j}(X) \nabla \mathrm{G}_{j}(X)=\mathbf{0},
$$

where $\mathbf{0}$ is a $p$-dimensional zero vector. We note $\mathrm{G} \equiv\left(\mathrm{G}_{j_{1}}, \ldots, \mathrm{G}_{j_{p-m}}\right)$ with $j_{s} \in I, s=1, \ldots, p-m$ and $p-m=|I|$. Since $\mathfrak{D G}(X)$ has full rank $p-m$ for $X \in \mathcal{U}$, its row vectors are linearly independent (D) stands for the derivative operator). Therefore $\sum_{j \in I} \mathrm{G}_{j}(X) \nabla \mathrm{G}_{j}(X)=\mathbf{0}$ if and only if the scalars $\mathrm{G}_{j}(X)=0$ for all $j \in I$. This is equivalent to the fact that $X \in \mathcal{L}$. Therefore, strict inequality $\langle\nabla \mathrm{E}(X) \mid \dot{X}\rangle<0$ holds for $X \in \mathcal{U} \backslash \mathcal{L}$ and hence the compact set $\mathcal{L}$ is asymptotically stable (see Appendix A for details).

\subsection{Explicit illustrations}

ExAmple 2.2 (Mixed canonical modulated-dissipative dynamics (MCMD)) Here, $p=2, m=1$ and $\mathrm{G}_{1}(x, y):=\mathrm{H}(x, y)-\mathrm{r}$, where $\mathrm{H}(x, y)$ is a Hamiltonian function on $\mathbb{R}^{2}$ into $\mathbb{R}_{\geqslant 0}$. Assume that the set $\mathcal{L}:=\left\{(x, y) \in \mathbb{R}^{2} \mid \mathrm{H}(x, y)-\mathrm{r}=0\right\}$ is a smooth, simple, closed curve in $\mathbb{R}^{2}$. For a given strictly positive (or negative) function $\mathrm{O}(x, y ; \Lambda)$, we define (without explicitly writing the parameters $\Lambda$ )

$$
\mathrm{D}(x, y)=\mathrm{O}(x, y)\left(\begin{array}{c}
-\frac{\partial \mathrm{H}}{\partial y}(x, y) \\
-\frac{\partial \mathrm{H}}{\partial x}(x, y)
\end{array}\right) \text {. }
$$

An MCMD is given as

$$
\begin{aligned}
& \dot{x}=-\mathrm{O}(x, y) \frac{\partial \mathrm{H}}{\partial y}(x, y)-(\mathrm{H}(x, y)-\mathrm{r}) \frac{\partial \mathrm{H}}{\partial x}(x, y), \\
& \dot{y}=-\mathrm{O}(x, y) \frac{\partial \mathrm{H}}{\partial x}(x, y)-(\mathrm{H}(x, y)-\mathrm{r}) \frac{\partial \mathrm{H}}{\partial y}(x, y) .
\end{aligned}
$$

This is a limit cycle oscillator with function $O$ playing the role of a clock with a local time scale on $\mathcal{L}$. As an illustration, consider $\mathrm{H}(x, y)=-\mathrm{d} \log (x)+\mathrm{e} x-\mathrm{a} \log (y)+$ by and $\mathrm{O}(x, y ; \Lambda)=\lambda x y$, both defined on the strictly positive quadrant $\mathbb{R}_{>0}^{2}$. Here, there is a single flow parameter $\Lambda=\{\lambda\}$, and the strictly positive geometric parameters are $\{a, b, d, e, r\}$. The resulting dynamics on the limit cycle $\mathcal{L}$ is described by the Lotka-Volterra equations

$$
\begin{aligned}
& \dot{x}=-\lambda(\mathrm{a} x-\mathrm{b} x y), \\
& \dot{y}=-\lambda(\mathrm{d} y-\mathrm{e} x y) .
\end{aligned}
$$

Example 2.3 (Mixed Canonical-Dissipative Dynamics (MCD)) The class of MCMD systems for which $\mathrm{O} \equiv \lambda$ (a constant) are known as mixed MCD. These dynamical systems have been considered for applications in Schweitzer et al. (2001) and Schweitzer (2003). As an illustration, consider the 


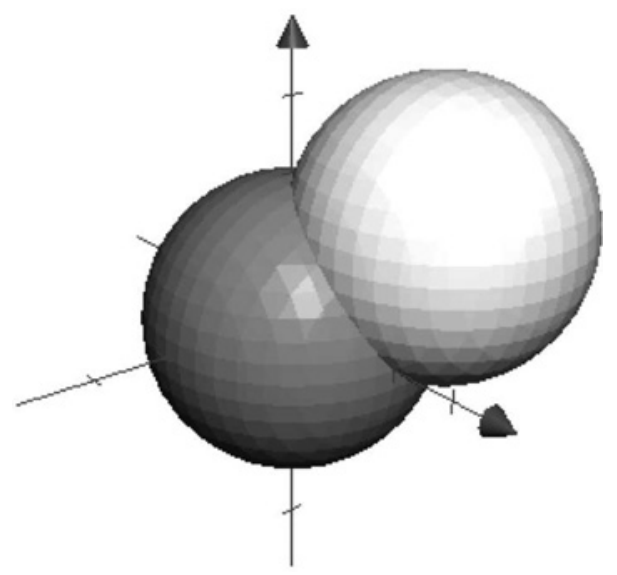

FIG. 1. Two spheres $\mathrm{G}_{1}(x, y, z):=x^{2}+y^{2}+z^{2}-1$ (dark gray) and $\mathrm{G}_{2}(x, y, z):=\left(x-\frac{2}{3}\right)^{2}+\left(y-\frac{2}{3}\right)^{2}+\left(z-\frac{2}{3}\right)^{2}-1$ (light gray).

Mathews-Lakshmanan oscillator presented in Mathews \& Lakshmanan (1974). ${ }^{3}$ The Hamiltonian is $\mathrm{H}(x, y)=\log (\cosh (y))+\frac{1}{2} \log \left(\mathrm{a}+x^{2}\right)$ with geometric parameter $\mathrm{a}>0$, and its dynamics reads as

$$
\begin{aligned}
& \dot{x}=-\lambda \tanh (y)-(H(x, y)-r) \frac{x}{\mathrm{a}+x^{2}}, \\
& \dot{y}=-\lambda \frac{x}{\mathrm{a}+x^{2}}-(\mathrm{H}(x, y)-\mathrm{r}) \tanh (y) .
\end{aligned}
$$

An orbit of the canonical dynamics (i.e. on $\mathcal{L}$ ) is explicitly given by

$$
\begin{aligned}
& x(t)=\sqrt{\exp (2 \mathrm{r})-\mathrm{a}} \sin \left(\frac{\lambda}{\exp (\mathrm{r})} t+\phi\right) \\
& y(t)=\tanh ^{-1}\left(\frac{\sqrt{\exp (2 \mathrm{r})-\mathrm{a}}}{\exp (\mathrm{r})} \cos \left(\frac{\lambda}{\exp (\mathrm{r})} t+\phi\right)\right) .
\end{aligned}
$$

Note that the solution exhibits an amplitude-dependent frequency, which is a characteristic of nonlinear oscillators.

${ }^{3}$ With $\lambda \sqrt{\mathrm{b}} s:=x$ and $\sqrt{\mathrm{b}} z=: \tanh (y)$, then $\dot{s}=z$ and the canonical dynamics become

$$
\left(1+\mathrm{f} s^{2}\right) \ddot{s}+\left(\alpha-f \dot{s}^{2}\right) s=0
$$

with $\mathrm{f}:=\mathrm{b}\left(\lambda^{2} / \mathrm{a}\right)$ and $\alpha:=\lambda^{2} / \mathrm{a}$. The oscillator is presented in this from in Mathews \& Lakshmanan (1974). 


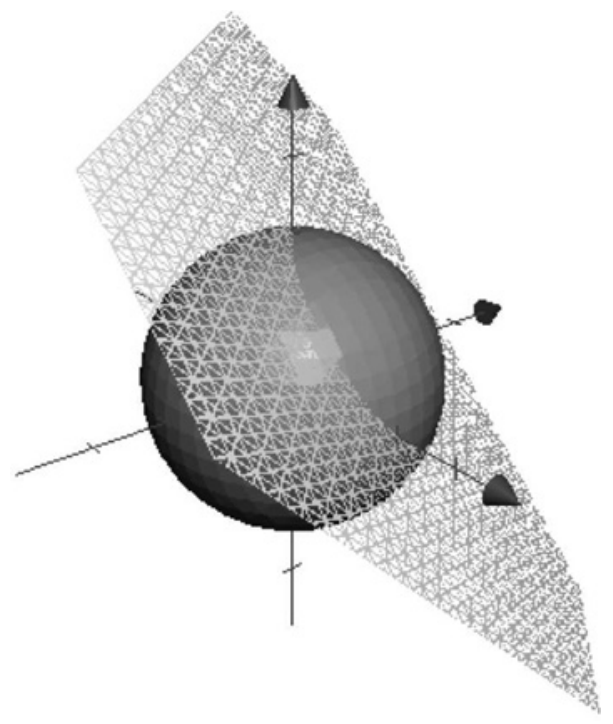

FIG. 2. Sphere $\mathrm{G}_{1}(x, y, z):=x^{2}+y^{2}+z^{2}-1$ (dark gray) and plane $\mathrm{G}_{2}(x, y, z):=x+y+z-1$ (light striped gray).

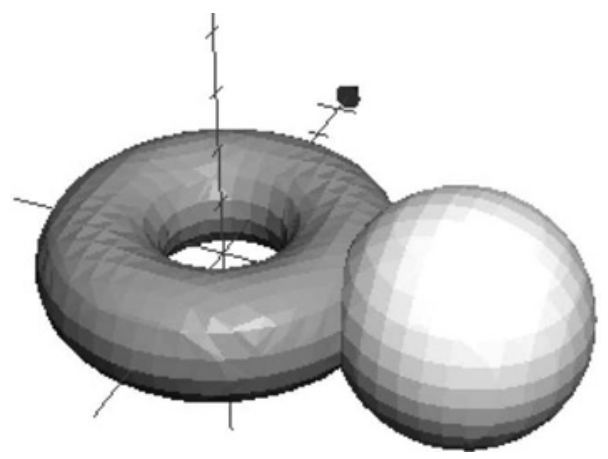

FIG. 3. Torus $\mathrm{G}_{1}(x, y, z):=\left(x^{2}+y^{2}+z^{2}+\frac{3}{4}\right)^{2}-4\left(x^{2}+y^{2}\right)$ (dark gray) and sphere $\mathrm{G}_{2}(x, y, z):=(x-2)^{2}+y^{2}+z^{2}-1($ light gray) with $r_{1}=1, r_{2}=\frac{1}{2}$ and $a=2$ values of the geometric parameters.

ExAmple 2.4 (Dynamics on $\mathbb{S}^{2}$ ) Here, $p=3, m=2$ and $\mathrm{G}_{1}(x, y, z):=x^{2}+y^{2}+z^{2}-1$. Hence, the attracting submanifold is $\mathbb{S}^{2}$. Due to the dimensionality of the attracting submanifold, several possibilities exist for $D$. For instance, with flow parameters $\Lambda:=\left\{\lambda_{1}, \lambda_{2}, \lambda_{3}\right\}$, we may have

$$
\begin{array}{cc}
\text { Euler equations } & \text { Linear solenoidal vector field } \\
\mathrm{D}(X ; \Lambda)=\left(\begin{array}{c}
\left(\lambda_{2}-\lambda_{3}\right) y z \\
\left(\lambda_{3}-\lambda_{1}\right) x z \\
\left(\lambda_{1}-\lambda_{2}\right) x y
\end{array}\right) & \mathrm{D}(X ; \Lambda)=\left(\begin{array}{ccc}
0 & \lambda_{1} & \lambda_{2} \\
-\lambda_{1} & 0 & \lambda_{3} \\
-\lambda_{2} & -\lambda_{3} & 0
\end{array}\right)\left(\begin{array}{l}
x \\
y \\
z
\end{array}\right) .
\end{array}
$$


EXAmple 2.5 (Dynamics on one-dimensional submanifold) Here, $p=3$ and $m=1$, and to create an attractor of dimension 1 we need two functions: $G_{1}$ and $G_{2}$. Let us exhibit three illustrations. For all cases, the vector $\mathrm{D}$ is uniquely determined, up to a constant and orientation.

(i) Sphere-Sphere

$\mathrm{G}_{1}(x, y, z):=x^{2}+y^{2}+z^{2}-1$ and $\mathrm{G}_{2}(x, y, z):=\left(x-\frac{2}{3}\right)^{2}+\left(y-\frac{2}{3}\right)^{2}+\left(z-\frac{2}{3}\right)^{2}-1$ (c.f. Fig. 1)

$$
\left(\begin{array}{l}
\dot{x} \\
\dot{y} \\
\dot{z}
\end{array}\right)=\lambda\left(\begin{array}{l}
y-z \\
z-x \\
x-y
\end{array}\right)-2 \mathrm{G}_{1}(x, y, z)\left(\begin{array}{l}
x \\
y \\
z
\end{array}\right)-2 \mathrm{G}_{2}(x, y, z)\left(\begin{array}{l}
x-\frac{2}{3} \\
y-\frac{2}{3} \\
z-\frac{2}{3}
\end{array}\right) .
$$

(ii) Sphere-Plane

$\mathrm{G}_{1}(x, y, z):=x^{2}+y^{2}+z^{2}-1$ and $\mathrm{G}_{2}(x, y, z):=x+y+z-1$ (c.f. Fig. 2)

$$
\left(\begin{array}{l}
\dot{x} \\
\dot{y} \\
\dot{z}
\end{array}\right)=\lambda\left(\begin{array}{l}
y-z \\
z-x \\
x-y
\end{array}\right)-2 \mathrm{G}_{1}(x, y, z)\left(\begin{array}{l}
x \\
y \\
z
\end{array}\right)-\mathrm{G}_{2}(x, y, z)\left(\begin{array}{l}
1 \\
1 \\
1
\end{array}\right) \text {. }
$$

For both cases (i) and (ii), all orbits converge towards a circle lying on the plane $\mathrm{G}_{2}(x, y, z):=x+$ $y+z-1$ with radius $\sqrt{\frac{2}{3}}$ and centre $\left(\frac{1}{3}, \frac{1}{3}, \frac{1}{3}\right)$. The orbit circulation on this attracting submanifold is identical for both examples. However, the transient dynamics (i.e. the convergence rate towards the attractor) explicitly depends on the choice of the $\mathrm{G}_{j}$ functions.

(iii) Torus-Sphere

$\mathrm{G}_{1}(x, y, z):=\left(x^{2}+y^{2}+z^{2}+\mathrm{r}_{1}^{2}-\mathrm{r}_{2}^{2}\right)^{2}-4 \mathrm{r}_{1}^{2}\left(x^{2}+y^{2}\right)$ (a torus centered at the origin) and $\mathrm{G}_{2}(x, y, z):=(x-\mathrm{a})^{2}+y^{2}+z^{2}-1$ (c.f. Fig. 3). The geometric parameters $\mathbf{r}_{1}, \mathbf{r}_{2}$ and a are chosen such that the intersection between the torus and the sphere forms a closed curve.

$$
\left(\begin{array}{c}
\dot{x} \\
\dot{y} \\
\dot{z}
\end{array}\right)=\lambda\left(\begin{array}{c}
-2 y z \mathrm{r}_{1}^{2} \\
z\left(g_{m}(x-\mathrm{a})-g_{p} x\right) \\
\mathrm{a} y g_{p}
\end{array}\right)-4 \mathrm{G}_{1}(x, y, z)\left(\begin{array}{c}
g_{p} x \\
g_{p} y \\
g_{m} z
\end{array}\right)-2 \mathrm{G}_{2}(x, y, z)\left(\begin{array}{c}
x-\mathrm{a} \\
y \\
z
\end{array}\right)
$$

with $g_{p}=x^{2}+y^{2}+z^{2}-\left(r_{2}^{2}+r_{1}^{2}\right)$ and with $g_{m}=x^{2}+y^{2}+z^{2}-\left(r_{2}^{2}-r_{1}^{2}\right)$.

\subsection{Coupling dynamics: $\mathrm{C}_{k}$}

Local dynamics are coupled via the gradient of a continuously differentiable positive semi-definite potential $\mathrm{V}(X) \geqslant 0$. Specifically, coupling dynamics are defined as

$$
\mathrm{C}_{k}(X):=-\mathrm{C}_{k} \frac{\partial \mathrm{V}}{\partial X_{k}}(X)
$$

with $X=\left(X_{1}, \ldots, X_{N}\right) \in \mathbb{R}^{p N}$ and coupling strengths $\mathrm{c}_{k}>0$. The function $\mathrm{V}$ depends on the entries $a_{k, j}$ of an $N \times N$ weighted adjacency matrix $A$ associated with a given connected and undirected networknote that additional parameters can also be introduced. To ensure the consistency of $\mathrm{V}$ with respect to the entries $a_{k, j}$ of $A$, we impose that if $a_{k, j}=0$ (and hence $a_{j, k}=0$, since the network is undirected), then the $p \times p$ dimensional matrix $\left(\partial^{2} \mathrm{~V} / \partial X_{j} \partial X_{k}\right)(X)=\mathbf{0}$. This ensures that only adjacent vertices interact. ${ }^{4}$

\footnotetext{
${ }^{4}$ Since $\mathrm{V}$ is twice continuously differentiable, it follows that interchanging the order of differentiation gives the same second partial derivatives and so $\left(\partial^{2} \mathrm{~V} / \partial X_{k} \partial X_{j}\right)(X)=\mathbf{0}$.
} 
We further suppose that

$$
\mathrm{V}(X)=0 \Longleftrightarrow X_{k}=X_{j} \quad \forall k, j
$$

with local state variables $X_{k}=\left(x_{k, 1}, \ldots, x_{k, p}\right) \in \mathbb{R}^{p}$. Any $X \in \mathbb{R}^{p N}$ satisfying Equations (2.3) is a minimum of $\mathrm{V}$, and therefore $\nabla \mathrm{V}(X)=\mathbf{0}$ (i.e. local systems with equal dynamical states do not interact).

For technical reasons, we will always suppose that $\mathrm{V}$ satisfies

$$
\forall X^{*} \in \mathcal{M},\left\langle X \mid \mathfrak{D}^{2} \vee\left(X^{*}\right) X\right\rangle=0 \Longleftrightarrow X_{k}=X_{j} \quad \forall k, j,
$$

where $\mathfrak{D}^{2} \mathrm{~V}\left(X^{*}\right)$ is the second total derivative (i.e. the Hessian) of $\mathrm{V}$ evaluated at $X^{*}$, and $\mathcal{M}$ is the consensual submanifold that is defined below.

Consensual Submanifold $\mathcal{M}$

Local attractors $\mathcal{L}_{k}$ only differ according to the choice of the functions $\mathrm{G}_{j}$. We will suppose that

$$
\mathcal{L}:=\bigcap_{k=1}^{N} \mathcal{L}_{k}=\left\{X \in \mathbb{R}^{p} \mid \mathrm{G}(X):=\left(\mathrm{G}_{j_{1}}(X), \ldots, \mathrm{G}_{j_{m}}(X)\right)=\mathbf{0} \in \mathbb{R}^{p-m}\right\}
$$

is an $m$-dimensional compact submanifold with $j_{s} \in \bigcup_{k=1}^{N} I_{k}, \quad s=1, \ldots, m$ and $m:=\left|\bigcup_{k=1}^{N} I_{k}\right|$. The set $\mathcal{L}$ is a local attractor shared by all local dynamics. This, together with Equations (2.3), enables us to define

$$
\mathcal{M}:=\left\{X \in \mathbb{R}^{p N} \mid X_{1} \in \mathcal{L} \text { and } X_{k}=X_{j} \forall k, j\right\},
$$

which is a $m$-dimensional compact submanifold of $\mathbb{R}^{p N}$ (see Appendix $\mathrm{C}$ for details), here called consensual submanifold. For any element $X \in \mathcal{M}, X_{k}=X_{j}$ for all $k, j$ and so, in particular, $X_{k}=X_{1}$. Hence, by the condition $X_{1} \in \mathcal{L}$, all local dynamics are to be found on $\mathcal{L}$.

EXAMPLE 2.6 (Laplacian potential) Let $L$ be a $N \times N$ Laplacian matrix ${ }^{5}$ associated to a connected and undirected network with positive adjacency entries (i.e. $a_{k, j}=a_{j, k} \geqslant 0$ for all $j, k$ ). The Laplacian potential is defined as

$$
\mathrm{V}(X)=\frac{1}{2} \sum_{j=1}^{p} \mathrm{a}_{j}\left\langle x_{j} \mid L x_{j}\right\rangle
$$

with $0<\mathrm{a}_{j}$ and $x_{j}:=\left(x_{1, j}, \ldots, x_{N, j}\right), j=1, \ldots, p$, and where $x_{k, j}$ is the $j$ th state variable of the $k$ th local dynamics. One can verify that $\mathrm{V}$ is positive semi-definite for which the equivalences in Equations (2.3) and (2.4) hold.

\subsection{Parametric dynamics: $\mathrm{P}_{k}$}

In this contribution, self-adaptivity is realized by allowing the flow parameters $\Lambda_{k}$ to have their own dynamics (i.e. letting the parameters be time-dependent: $\Lambda_{k} \rightsquigarrow \Lambda_{k}(t)$ ). Their rate of change $\dot{\Lambda}_{k}(t)$ is determined by the parametric dynamics $\mathrm{P}_{k}$ for $k=1, \ldots, N$. Hence $\Lambda_{k}(t)$ acquire the status of variables of the global dynamical system. Later, they will be called flow parametric variables (f-PV). In the rest of this section, we consider homogeneous dynamics $\mathrm{L}_{k} \equiv \mathrm{L}$ (i.e. $\left|\Lambda_{k}\right|=q \forall k$ ). The heterogeneous case that we focus on is straightforwardly generalized (refer to Section 3.4).

\footnotetext{
5 A Laplacian matrix $L$ is defined as $L:=D-A$ where $D$ is the diagonal matrix with entries $d_{k, k}:=\sum_{j=1}^{N} a_{k, j}$ and $a_{k, j}$ are the entries of the adjacency matrix $A$.
} 
By suitably constructing the parametric dynamics $\mathrm{P}_{k}$, the $\Lambda_{k}(t)$ will ultimately converge towards a common and constant set $\Lambda_{c}$ (i.e. $\lim _{t \rightarrow \infty} \Lambda_{k}(t)=\Lambda_{\mathrm{c}}$ for $k=1, \ldots, N$ ). We now discuss the convergence issues and the resulting limits.

Convergence-The goal is to find control functions $\mathrm{P}_{k}(X)$ for which orbits of Equations (2.1) satisfy

$$
\lim _{t \rightarrow \infty} \Lambda_{k}(t)=\lim _{t \rightarrow \infty} \Lambda_{\mathrm{c}}+\epsilon_{k}(t)=\Lambda_{\mathrm{c}} \quad k=1, \ldots, N
$$

The mismatches $\epsilon_{k}$ play the role of perturbations around the consensual parameter set $\Lambda_{\mathrm{c}}$.

Limit values-While the value of the limit set $\Lambda_{\mathrm{c}}$ is generally unknown, our class of dynamics allows an explicit calculation of $\Lambda_{\mathrm{c}}$. Consider orbits $\left(\Lambda_{1}(t), \ldots, \Lambda_{N}(t)\right)$ solving Equations (2.1b), and let us construct functions $\mathrm{P}_{k}$ for which (i) Equations (2.7) hold and (ii) there exists an $\mathbb{R}^{q}$-valued function $\mathrm{J}\left(\Lambda_{1}, \ldots, \Lambda_{N}\right)$ such that

$$
\frac{\mathrm{d}\left[\mathrm{J}\left(\Lambda_{1}(t), \ldots, \Lambda_{N}(t)\right)\right]}{\mathrm{d} t}=\mathbf{0} \quad \forall t \geqslant 0 \Longleftrightarrow \mathrm{J}\left(\Lambda_{1}(t), \ldots, \Lambda_{N}(t)\right)=\mathbf{C} \quad \forall t \geqslant 0,
$$

where $\mathbf{C}$ is a $q$-dimensional constant vector determined by the initial values $\left(\Lambda_{1}(0), \ldots, \Lambda_{N}(0)\right)$. In other words, $\mathrm{J}$ is a constant of motion of the dynamics and we therefore have

$$
\lim _{t \rightarrow \infty} \mathrm{J}\left(\Lambda_{1}(t), \ldots, \Lambda_{N}(t)\right)=\mathrm{J}\left(\Lambda_{\mathrm{c}}, \ldots, \Lambda_{\mathrm{c}}\right)=\mathbf{C} .
$$

Equation (2.8) is a system of $q$ unknowns with $q$ equations, which can now be solved.

2.4.1 Dynamics of flow parametric variables. To get insights into the role played by the flow parametric variables, we first focus on systems of dimension 2 (i.e. MCD oscillators), all having the same potential (i.e. $\mathrm{E}_{k} \equiv \frac{1}{2} \mathrm{G}^{2}$ for all $k$ ). In this case, for a given $\mathrm{G}, \mathrm{D}_{k} \equiv \lambda_{k} \mathrm{~K}$ is uniquely determined up to a multiplicative factor (here, a constant $\lambda_{k}$ )

$$
\mathrm{D}\left(X_{k} ; \lambda_{k}\right)=\lambda_{k} \mathrm{~K}\left(X_{k}\right):=\lambda_{k}\left(\begin{array}{c}
-\frac{\partial \mathrm{G}}{\partial y}\left(X_{k}\right) \\
-\frac{\partial \mathrm{G}}{\partial x}\left(X_{k}\right)
\end{array}\right)=\left(\begin{array}{cc}
0 & \lambda_{k} \\
-\lambda_{k} & 0
\end{array}\right)\left(\begin{array}{l}
\frac{\partial \mathrm{G}}{\partial x}\left(X_{k}\right) \\
\frac{\partial \mathrm{G}}{\partial y}\left(X_{k}\right)
\end{array}\right),
$$

with $X_{k}=\left(x_{k}, y_{k}\right)$. Here, $\lambda_{k}$ controls the angular velocity of the $k$ th local dynamics on its attractor (i.e. closed curve in $\mathbb{R}^{2}$ ). We now assign to $\lambda_{k}$ the role of a parametric variable (i.e. $\lambda_{k} \rightsquigarrow \lambda_{k}(t)$ ) with dynamics defined as

$$
\dot{\lambda}_{k}=-\mathrm{s}_{k}\left\langle\left(\begin{array}{l}
\frac{\partial \mathrm{V}}{\partial x_{k}}(X) \\
\frac{\partial \mathrm{V}}{\partial y_{k}}(X)
\end{array}\right) \mid\left(\begin{array}{c}
-\frac{\partial \mathrm{G}}{\partial y}\left(X_{k}\right) \\
-\frac{\partial \mathrm{G}}{\partial x}\left(X_{k}\right)
\end{array}\right)\right\rangle=-\mathrm{s}_{k}\left\langle\frac{\partial \mathrm{V}}{\partial X_{k}}(X) \mid \mathrm{K}\left(X_{k}\right)\right\rangle,
$$

where $0<\mathrm{s}_{k}$ are given susceptibility constants. For $\mathrm{S}_{k} \gg 1$, the $k$ th oscillator is strongly influenced by its neighbour, whereas $\mathbf{s}_{k} \ll 1$ reflects its 'stubbornness' to adapt. If, in the extreme case, $\mathbf{s}_{k}=0, \lambda_{k}(t)$ becomes a constant and thus recovers its original status of a fixed and constant parameter. 
If we suppose that $\sum_{k=1}^{N}\left\langle\left(\partial \mathrm{V} / \partial X_{k}\right)(X) \mid \mathrm{K}\left(X_{k}\right)\right\rangle=0$ for all $X$, we have the following constant of motion

$$
\mathrm{J}\left(\lambda_{1}, \ldots, \lambda_{N}\right):=\sum_{k=1}^{N} \frac{\lambda_{k}}{\mathrm{~s}_{k}}
$$

Indeed, for $\lambda_{k}(t)(k=1, \ldots, N)$ orbits of Equations (2.10), we have

$$
\frac{\mathrm{d}\left[\mathrm{J}\left(\lambda_{1}(t), \ldots, \lambda_{N}(t)\right)\right]}{\mathrm{d} t}=\sum_{k=1}^{N} \frac{\dot{\lambda}_{k}(t)}{\mathrm{s}_{k}}=\sum_{k=1}^{N} \frac{-\mathrm{s}_{k}}{\mathrm{~s}_{k}}\left\langle\frac{\partial \mathrm{V}}{\partial X_{k}}(X) \mid \mathrm{K}\left(X_{k}\right)\right\rangle=0 .
$$

It is worth emphasizing that the constant of motion $\mathrm{J}$ only depends on the f-PV $\lambda_{k}$ and on the susceptibility constants $\mathrm{s}_{k}$, but not on the adjacency matrix $A$. Therefore, if a consensual and common $\lambda_{\mathrm{c}}$ is reached (i.e. convergence in (2.7) holds), its value does not depend on the topology of the network.

As an example, consider the case where $\mathrm{G}(X)=x^{2}+y^{2}-\mathrm{r}$ and the coupling dynamics is given by a Laplacian potential defined as $\mathrm{V}(X)=\langle x \mid L x\rangle+\langle y \mid L y\rangle$ with $x=\left(x_{1}, \ldots, x_{N}\right)$ (idem for $y$ ). In this case, we obtain

$$
\sum_{j=1}^{N} l_{k, j} \frac{\partial \mathrm{G}}{\partial x}\left(X_{j}\right)=\frac{\partial \mathrm{V}}{\partial x_{k}}(X) \text { and } \sum_{j=1}^{N} l_{k, j} \frac{\partial \mathrm{G}}{\partial y}\left(X_{j}\right)=\frac{\partial \mathrm{V}}{\partial y_{k}}(X)
$$

and hence

$$
\sum_{k=1}^{N}\left\langle\frac{\partial \mathrm{V}}{\partial X_{k}}(X) \mid \mathrm{K}\left(X_{k}\right)\right\rangle=\sum_{k=1}^{N} \sum_{j=1}^{N} l_{k, j}\left(\frac{\partial \mathrm{G}}{\partial x}\left(X_{j}\right) \frac{\partial \mathrm{G}}{\partial y}\left(X_{k}\right)-\frac{\partial \mathrm{G}}{\partial y}\left(X_{j}\right) \frac{\partial \mathrm{G}}{\partial x}\left(X_{k}\right)\right)=0
$$

since, by Lemma B.1 in Appendix B, the last equality is zero.

GENERALIZATION FOR HIGHER DIMENSIONS AND MUlTi $f$-PV. In Equations (2.9), the first equality (vector representation) naturally extends to higher dimensions in the case involving single adapting parameters. The matrix representation (second equality) in Equations (2.9) enables generalizations to the multiparametric cases. Let us detail these two situations for homogeneous O-G systems (i.e. $\mathrm{D}_{k} \equiv \mathrm{D}$ for all $k$ ).

Single adapting flow parameter. By construction of the local dynamics, D can always be written as $\mathrm{D}\left(X_{k}, \lambda_{k}\right):=\lambda_{k} \mathrm{~K}\left(X_{k}\right)$ and so, for this case, the set of $\mathrm{f}-\mathrm{PV}$ is reduced to a singleton $\lambda_{k}$. For arbitrary dimension $p$, a natural generalization for the parametric dynamics will be

$$
\dot{\lambda}_{k}=-\mathrm{s}_{k}\left\langle\frac{\partial \mathrm{V}}{\partial X_{k}}(X) \mid \mathrm{K}\left(X_{k}\right)\right\rangle .
$$

If we suppose that

$$
\sum_{k=1}^{N}\left\langle\frac{\partial \mathrm{V}}{\partial X_{k}}(X) \mid \mathrm{K}\left(X_{k}\right)\right\rangle=0 \quad \forall X
$$

we then have the constant of motion as in Equation (2.11). Hence, a single constant of motion $\mathrm{J}$ depending on $\lambda_{k}$ and $s_{k}$ determines the asymptotic value $\lambda_{\mathrm{c}}$ (i.e. the consensual value is independent of the network topology). Note that $\left(\partial \mathrm{V} / \partial X_{k}\right)(X)$ and $\mathrm{K}\left(X_{k}\right)$ can be seen as generalized forces and, respectively, displacements. Hence the orthogonality in Equation (2.12) expresses the fact that no mechanical work due to the network forces is produced. 
Multi-adapting flow parameters. Here we focus on cases where $\mathrm{D}$ can be written as $\mathrm{D}\left(X_{k}, \Lambda_{k}\right):=$ $T_{k} \mathrm{~K}\left(X_{k}\right)$ with $T_{k}$ being a $p \times p$ anti-symmetric matrix. The upper diagonal elements of $T_{k}$ are given ${ }^{6}$ by the f-PV $\Lambda_{k} \in \mathbb{R}^{p(p-1) / 2}$. This is compatible with O-G dynamics, provided the orthogonality between $T_{k}$ and $\mathrm{K}$ is ensured, namely

$$
\left\langle\mathrm{D}\left(X_{k}, \Lambda_{k}\right) \mid \nabla \mathrm{E}_{k}(X)\right\rangle=\left\langle T_{k} \mathrm{~K}\left(X_{k}\right) \mid \sum_{j \in I_{k}} \mathrm{G}_{j}\left(X_{k}\right) \nabla \mathrm{G}_{j}\left(X_{k}\right)\right\rangle=0 .
$$

In particular, when $I_{k}:=\{1\}$ for all $k$ (refer to Section 2.1), this holds for $\mathrm{K}\left(X_{k}\right):=\nabla \mathrm{G}_{1}\left(X_{k}\right)$ and $T_{k}$ any anti-symmetric matrix (see Lemma B.2 in Appendix B for details). Inspired by the matrix representation in Equations (2.9), a generalized multi parametric dynamics can be constructed as

$$
\dot{\lambda}_{k, l, s}=-\mathrm{s}_{k, l, s}\left\langle\left(\begin{array}{c}
\frac{\partial \mathrm{V}}{\partial x_{k, l}}(X) \\
\frac{\partial \mathrm{V}}{\partial x_{k, s}}(X)
\end{array}\right) \mid\left(\begin{array}{c}
-\frac{\partial \mathrm{G}_{1}}{\partial x_{s}}\left(X_{k}\right) \\
-\frac{\partial \mathrm{G}_{1}}{\partial x_{l}}\left(X_{k}\right)
\end{array}\right)\right\rangle k=1, \ldots, N, \quad l, s=1, \ldots, p, \quad s>l,
$$

where $\lambda_{k, l, s}$ are the $l$ th row, sth column entries of the $k$ th matrix $T_{k}$.

As far as constants of motion are concerned, the assumption that for all $s$ and $l(l, s=1 \ldots, p$, and $s>l$ ), we have

$$
\sum_{k=1}^{N}\left\langle\left(\begin{array}{l}
\frac{\partial \mathrm{V}}{\partial x_{k, l}}(X) \\
\frac{\partial \mathrm{V}}{\partial x_{k, s}}(X)
\end{array}\right) \mid\left(\begin{array}{c}
-\frac{\partial \mathbf{G}_{1}}{\partial x_{s}}\left(X_{k}\right) \\
-\frac{\partial \mathbf{G}_{1}}{\partial x_{l}}\left(X_{k}\right)
\end{array}\right)\right\rangle=0 \quad \forall X
$$

ensures that $p(p-1) / 2$ constants of motion exist. These are given by $J_{l, s}\left(\lambda_{1, l, s}, \ldots, \lambda_{N, l, s}\right):=$ $\sum_{k=1}^{N}\left(\lambda_{k, l, s} / \mathbf{s}_{k, l, s}\right)$. Again, if a consensual and common $\lambda_{c, l, s}$ is reached, its value does not depend on the topology of the network (since the function $\mathrm{J}_{l, s}$ only depends on $\lambda_{k, l, s}$ and $\mathbf{s}_{k, l, s}$ ).

REMARK For both cases (single- and multi-adapting flow parameters), Equation (2.12), respectively, Equation (2.13), imply that

$$
\langle\nabla \mathrm{V}(X) \mid \overrightarrow{\mathrm{D}}(X)\rangle=0 \quad \forall X,
$$

with $\quad \overrightarrow{\mathrm{D}}(X):=\left(\lambda \mathrm{K}\left(X_{1}\right), \ldots, \lambda \mathrm{K}\left(X_{N}\right)\right) \quad$ (constant scalar $\left.\quad \lambda\right), \quad$ respectively, $\quad \overrightarrow{\mathrm{D}}(X):=$ $\left(T \nabla \mathrm{G}_{1}\left(X_{1}\right), \ldots, T \nabla \mathrm{G}_{1}\left(X_{N}\right)\right)$ (constant anti-symmetric matrix $\left.T\right)$. With the orthogonality property in Equation (2.14), the global dynamical system is itself an $\mathrm{O}-\mathrm{G}$ system. This expresses once again a non-working characteristic of the network forces.

${ }^{6}$ From $\Lambda_{k}=\left(\lambda_{k, 1,2}, \ldots, \lambda_{k, 1, p}, \lambda_{k, 2,3}, \ldots, \lambda_{k, 2, p}, \ldots, \lambda_{k, p-1, p}\right) \in \mathbb{R}^{p(p-1) / 2}$, we define $T_{k}$ as

$$
\left(\begin{array}{ccccc}
0 & \lambda_{k, 1,2} & \ldots & \ldots & \lambda_{k, 1, p} \\
-\lambda_{k, 1,2} & 0 & \lambda_{k, 2,3} & \ldots & \lambda_{k, 2, p} \\
\vdots & -\lambda_{k, 2,3} & \ddots & & \vdots \\
\vdots & \vdots & & \ddots & \lambda_{k, p-1, p} \\
-\lambda_{k, 1, p} & -\lambda_{k, 2, p} & \ldots & \ldots & 0
\end{array}\right)
$$




\section{Network dynamics}

In this section, we first discuss the dynamics of interacting homogeneous local dynamics (i.e. $\mathrm{D}_{k} \equiv \mathrm{D}$ for all $\left.k\right)^{7}$ with constant (i.e trivial parametric dynamics $\mathrm{P}_{k} \equiv 0$ in Equations (2.1b)) and identical parameters (i.e. $\Lambda_{k}=\Lambda_{\mathrm{c}}$ for all $k$ ) (we refer the reader to Section 3.1). We then consider a network of homogeneous local dynamics with single (we refer the reader to Section 3.2) and multi (refer to Section 3.3) adapting parameters. Finally, we focus on heterogeneous dynamical systems with parametric dynamics (we refer the reader to Section 3.4).

\subsection{Network of homogeneous local dynamics with constant identical parameters}

Consider the dynamical System (2.1a) where all vertices are endowed with homogeneous local dynamics (i.e. $\mathrm{D}_{k} \equiv \mathrm{D}$ for all $k$ ). Here we suppose that $\mathrm{P}_{k} \equiv 0$ in Equations (2.1b). Therefore, local dynamics have a constant set of parameters which we suppose to be common to all local systems (i.e. $\Lambda_{k}=\Lambda_{\mathrm{c}}$ for all $k$ ). Let us discuss the existence of a consensual state and the convergence towards it.

Existence of a consensual state. The existence of a consensual state for System (2.1a) $\left(\mathrm{P}_{k} \equiv 0\right.$ in Equations (2.1b)) is guaranteed if initial conditions are in $\mathcal{M}$ (cf. (2.6)).

Convergence towards a consensual state. The convergence towards a consensual state is established by Lemma 3.1. For ease of notation, we remove the explicit dependence of $\Lambda_{\mathrm{c}}$.

LemMa 3.1 Suppose that

$$
\sum_{k=1}^{N}\left\langle\frac{\partial \mathrm{V}}{\partial X_{k}}(X) \mid \mathrm{D}\left(X_{k}\right)\right\rangle \leqslant 0 \quad \forall X
$$

Then there exists a set $\mathcal{U} \supset \mathcal{M}$ such that all orbits solving System $(2.1 \mathrm{a})^{8}$ with initial conditions in $\mathcal{U}$ converge towards $\mathcal{M}$.

Proof. The convergence towards $\mathcal{M}$ follows from Lyapunov's second method with Lyapunov function

$$
\mathrm{F}(X)=\sum_{k=1}^{N} \frac{1}{\mathrm{c}_{k}} \mathrm{E}_{k}\left(X_{k}\right)+\mathrm{V}(X) \geqslant 0
$$

\footnotetext{
${ }^{7}$ For compatibility with O-G systems, it is implicitly supposed that $\left\langle\mathrm{D} \mid \nabla \mathrm{E}_{k}\right\rangle=0$ for all $k$.

${ }^{8}$ Here, $\mathrm{P}_{k} \equiv 0$ in Equations (2.1b) and $\Lambda_{k}:=\Lambda_{\mathrm{c}}$ for all $k$.
} 
By construction, we have $\mathcal{M}=\left\{X \in \mathbb{R}^{p N} \mid \mathrm{F}(X)=0\right\}$. Computing the time derivative

$$
\begin{aligned}
\langle\nabla \mathrm{F}(X) \mid \dot{X}\rangle= & \sum_{k=1}^{N}\left\langle\frac{1}{\mathrm{c}_{k}} \nabla \mathrm{E}_{k}\left(X_{k}\right)+\frac{\partial \mathrm{V}}{\partial X_{k}}(X) \mid \mathrm{D}\left(X_{k}\right)-\nabla \mathrm{E}_{k}\left(X_{k}\right)-\mathrm{c}_{k} \frac{\partial \mathrm{V}}{\partial X_{k}}(X)\right\rangle \\
= & \sum_{k=1}^{N} \frac{1}{\mathrm{c}_{k}} \underbrace{\left\langle\nabla \mathrm{E}_{k}\left(X_{k}\right) \mid \mathrm{D}\left(X_{k}\right)\right\rangle}_{=0 \forall k}+\sum_{k=1}^{N} \underbrace{\left\langle\frac{\partial \mathrm{V}}{\partial X_{k}}(X) \mid \mathrm{D}\left(X_{k}\right)\right\rangle}_{\leqslant 0} \\
& \underbrace{-\sum_{k=1}^{N} \mathrm{c}_{k}\left\|\frac{1}{\mathrm{c}_{k}} \nabla \mathrm{E}_{k}\left(X_{k}\right)+\frac{\partial \mathrm{V}}{\partial X_{k}}(X)\right\|^{2}}_{\leqslant 0} .
\end{aligned}
$$

We now need to show that there exists $\mathcal{U} \supset \mathcal{M}$ such that the strict negativity $\langle\nabla \mathrm{F}(X) \mid \dot{X}\rangle<0$ holds for all $X \in \mathcal{U} \backslash \mathcal{M}$. For this, we show that there exists $\mathcal{U} \supset \mathcal{M}$ such that $-\sum_{k=1}^{N} \mathrm{c}_{k} \|\left(1 / \mathrm{c}_{k}\right) \nabla \mathrm{E}_{k}\left(X_{k}\right)+$ $\left(\partial \mathrm{V} / \partial X_{k}\right)(X) \|^{2}<0$ for all $X \in \mathcal{U} \backslash \mathcal{M}$. Remark that

$$
\sum_{k=1}^{N} \mathrm{c}_{k}\left\|\frac{1}{\mathrm{c}_{k}} \nabla \mathrm{E}_{k}\left(X_{k}\right)+\frac{\partial \mathrm{V}}{\partial X_{k}}(X)\right\|^{2}=0 \Longleftrightarrow \nabla \mathrm{F}(X)=\mathbf{0} .
$$

Therefore, we have to show that there exists $\mathcal{U} \supset \mathcal{M}$ such that $\nabla \mathrm{F}(X) \neq \mathbf{0}$ for all $X \in \mathcal{U} \backslash \mathcal{M}$. For this, we invoke Corollary E.2 in Appendix E. To apply Corollary E.2, we need to verify that, for all $X^{*} \in \mathcal{M}$, the kernel $\mathbb{k e r}\left(\mathfrak{D}^{2} \mathrm{~F}\left(X^{*}\right)\right)$ of the $p N \times p N$ Hessian $\mathfrak{D}^{2} \mathrm{~F}\left(X^{*}\right)$ is equal to the $\operatorname{kernel} \mathbb{k e r}\left(\mathfrak{D M}\left(X^{*}\right)\right)$ of the submanifold $\mathcal{M}$. This is done in Appendix $\mathrm{D}$ and concludes the proof.

\subsection{Network of homogeneous local dynamics with single adapting flow parameters}

Let the set of f-PV be reduced to a single element: $\lambda_{k}$. Define the local dynamics of the network as

$$
\mathrm{D}\left(X_{k}, \lambda_{k}\right):=\lambda_{k} \mathrm{~K}\left(X_{k}\right) \quad \text { and } \quad \mathrm{E}_{k}\left(X_{k}\right):=\frac{1}{2} \sum_{j \in I_{k}} \mathrm{G}_{j}\left(X_{k}\right)^{2} .
$$

The dynamical system is

$$
\begin{aligned}
\dot{X}_{k} & =\underbrace{\lambda_{k} \mathrm{~K}\left(X_{k}\right)-\sum_{j \in I_{k}} \mathrm{G}_{j}\left(X_{k}\right) \nabla \mathrm{G}_{j}\left(X_{k}\right)}_{\text {local dynamics }}-\underbrace{\mathrm{c}_{k} \frac{\partial \mathrm{V}}{\partial X_{k}}(X)}_{\text {coupling dynamics }} k=1, \ldots, N . \\
\dot{\lambda}_{k} & =\underbrace{-\mathrm{s}_{k}\left\langle\frac{\partial \mathrm{V}}{\partial X_{k}}(X) \mid \mathrm{K}\left(X_{k}\right)\right\rangle}_{\text {parametric dynamics }}
\end{aligned}
$$

Let us discuss the existence of a consensual state and the convergence towards it.

Existence of a consensual state. The existence of a consensual state for System (3.1) is guaranteed if initial conditions are in $\mathcal{M}$ (cf. (2.6)) and $\lambda_{k}:=\lambda_{\mathrm{c}}$ for all $k$ with $\lambda_{\mathrm{c}}$ a given constant. 
Convergence towards a consensual state. The convergence towards a consensual state as well as the explicit value of $\lambda_{c}$ are established by Proposition 3.2.

Proposition 3.2 Suppose that

$$
\sum_{k=1}^{N}\left\langle\frac{\partial \mathrm{V}}{\partial X_{k}}(X) \mid \mathrm{K}\left(X_{k}\right)\right\rangle=0 \quad \forall X
$$

Then there exists a set $\mathcal{U} \supset \mathcal{C}_{\lambda_{\mathrm{c}}}$ such that all orbits solving System (3.1) with initial conditions in $\mathcal{U}$ converge towards $\mathcal{C}_{\lambda_{\mathrm{c}}}$ with

$$
\mathcal{C}_{\lambda_{\mathrm{c}}}:=\left\{(X, \Lambda) \in \mathbb{R}^{N p} \times \mathbb{R}^{N} \mid X \in \mathcal{M} \text { and } \Lambda=\lambda_{\mathrm{c}} \mathbf{1}\right\} \quad \text { and } \quad \lambda_{\mathrm{c}}:=\frac{\sum_{k=1}^{N}\left(\lambda_{k}(0) / \mathrm{s}_{k}\right)}{\sum_{k=1}^{N}\left(1 / \mathrm{s}_{k}\right)}
$$

where $\mathbf{1}$ is an $N$-dimensional vector of 1 .

Proof. We refer the reader to Appendix F for the proof. 
EXAMPLE 3.3 Take $p=3$ and the attracting submanifold to be a closed curve in $\mathbb{R}^{3}$ (i.e. $m=1$ ). Select $\mathrm{G}_{1}$ and $\mathrm{G}_{2}$ functions as

$$
\mathrm{G}_{1}\left(X_{k}\right):=\mathrm{a} x_{k}^{2}+\mathrm{b} y_{k}^{2}+\mathrm{d} z_{k}^{2}-1 \text { and } \mathrm{G}_{2}\left(X_{k}\right):=\mathrm{a} x_{k}+\mathrm{b} y_{k}+\mathrm{d} z_{k}-1
$$

with $X_{k}=\left(x_{k}, y_{k}, z_{k}\right)$ and geometric parameters $\{\mathrm{a}, \mathrm{b}, \mathrm{d}\}$. The potentials are all identical: $\mathrm{E}_{k} \equiv \frac{1}{2}\left(\mathrm{G}_{1}^{2}+\right.$ $\mathrm{G}_{2}^{2}$ ). The vector product between $\nabla \mathrm{G}_{1}$ and $\nabla \mathrm{G}_{2}$ determines $\mathrm{D}$, which reads

$$
\mathrm{D}\left(X_{k}, \lambda_{k}\right)=\lambda_{k} \mathrm{~K}\left(X_{k}\right):=\lambda_{k}\left(\begin{array}{l}
\operatorname{bd}\left(y_{k}-z_{k}\right) \\
\operatorname{ad}\left(z_{k}-x_{k}\right) \\
\operatorname{ab}\left(x_{k}-y_{k}\right)
\end{array}\right),
$$

where the $\mathrm{f}-\mathrm{PV}$ is $\lambda_{k}$ and $\mathrm{a}, \mathrm{b}$ and $\mathrm{d}$ are geometric parameters. The coupling dynamics here is given by the Laplacian potential (refer to Example 2.6)

$$
\mathrm{V}(X):=\frac{1}{2}(\mathrm{a}\langle x \mid L x\rangle+\mathrm{b}\langle y \mid L y\rangle+\mathrm{d}\langle z \mid L z\rangle)
$$

with $x=\left(x_{1}, \ldots, x_{N}\right)$ (idem for $y$ and $z$ ). The orthogonality condition of Proposition 3.2 is satisfied as

$$
\begin{aligned}
\sum_{k=1}^{N}\left\langle\frac{\partial \mathrm{V}}{\partial X_{k}}(X) \mid \mathrm{K}\left(X_{k}\right)\right\rangle= & \sum_{k=1}^{N}\left\langle\left(\begin{array}{c}
\sum_{j=1}^{N} l_{k, j} \mathrm{a} x_{j} \\
\sum_{j=1}^{N} l_{k, j} \mathrm{~b} y_{j} \\
\sum_{j=1}^{N} l_{k, j} \mathrm{~d} z_{j}
\end{array}\right) \mid\left(\begin{array}{c}
\mathrm{bd}\left(y_{k}-z_{k}\right) \\
\mathrm{ad}\left(z_{k}-x_{k}\right) \\
\mathrm{ab}\left(x_{k}-y_{k}\right)
\end{array}\right)\right\rangle \\
= & \mathrm{d} \sum_{k=1}^{N} \sum_{j=1}^{N} l_{k, j}\left(\mathrm{a} x_{j} \mathrm{~b} y_{k}-\mathrm{b} y_{j} \mathrm{a} x_{k}\right)+\mathrm{b} \sum_{k=1}^{N} \sum_{j=1}^{N} l_{k, j}\left(\mathrm{~d} z_{j} \mathrm{a} x_{k}-\mathrm{ax} x_{j} \mathrm{~d} z_{k}\right) \\
& +\mathrm{a} \sum_{k=1}^{N} \sum_{j=1}^{N} l_{k, j}\left(\mathrm{~b} y_{j} \mathrm{~d} z_{k}-\mathrm{d} z_{j} \mathrm{~b} y_{k}\right)=0,
\end{aligned}
$$

since, by Lemma B.1 in Appendix B, the three terms in the last equality are zero, respectively.

For $k=1, \ldots, N$, the resulting dynamical system is

$$
\left(\begin{array}{c}
\dot{x}_{k} \\
\dot{y}_{k} \\
\dot{z}_{k}
\end{array}\right)=\underbrace{\lambda_{k}\left(\begin{array}{c}
\mathrm{bd}\left(y_{k}-z_{k}\right) \\
\mathrm{ad}\left(z_{k}-x_{k}\right) \\
\mathrm{ab}\left(x_{k}-y_{k}\right)
\end{array}\right)-2 \mathrm{G}_{1}\left(X_{k}\right)\left(\begin{array}{c}
\mathrm{a} x_{k} \\
\mathrm{~b} y_{k} \\
\mathrm{~d} z_{k}
\end{array}\right)-\mathrm{G}_{2}\left(X_{k}\right)\left(\begin{array}{l}
\mathrm{a} \\
\mathrm{b} \\
\mathrm{d}
\end{array}\right)}_{\text {local dynamics }}-\underbrace{\mathrm{c}_{k} \sum_{\sum_{j=1}^{N} l_{k, j} \mathrm{~b} y_{j}}^{\left(\sum_{j=1}^{N} l_{k, j} \mathrm{a} x_{j}\right.}}_{\text {coupling dynamics }}
$$




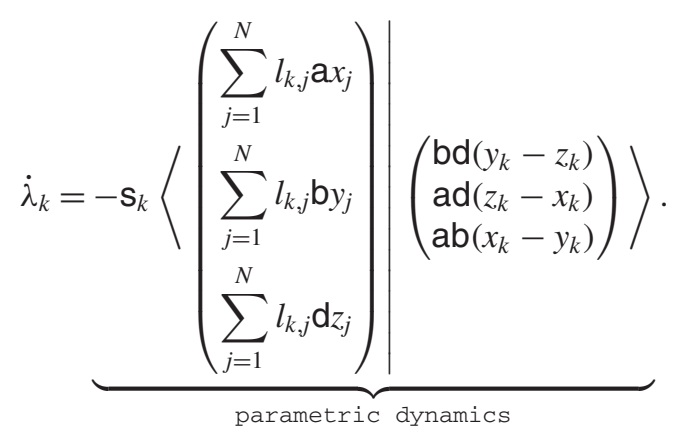

Although $A$ is symmetric, the heterogeneous coupling strengths $\mathrm{C}_{k}$ confer a weighted character to the (undirected) network (see Motter et al., 2005 and Chavez et al., 2005 for similar situations). As this also holds for the susceptibility constants $\mathbf{s}_{k}$, the resulting dynamics effectively involves two networks: one for the state variable interactions $\left(c_{k}\right.$ mediated) and the other for the adaptive mechanisms themselves ( $\mathrm{s}_{k}$ mediated).

\subsection{Network of homogeneous local dynamics with multi-adapting flow parameters}

We now present a class of network dynamics for the second type of generalization discussed in Section 2.4.1. Let the set $\Lambda_{k} \in \mathbb{R}^{p(p-1) / 2}$ of f-PV define a $p \times p$ anti-symmetric matrix $T_{k}$. Define the local dynamics of the network as

$$
\mathrm{D}\left(X_{k}, \Lambda_{k}\right):=T_{k} \nabla \mathrm{G}_{1}\left(X_{k}\right) \text { and } \mathrm{E}\left(X_{k}\right):=\frac{1}{2} \mathrm{G}_{1}\left(X_{k}\right)^{2},
$$

where we drop the index $k$ from the potentials $E$ since they are all identical. Note that $D$ is orthogonal to $\nabla \mathrm{E}$ (see Lemma B.2 in Appendix B for details). The dynamical system is

$$
\begin{aligned}
& \dot{X}_{k}=\underbrace{T_{k} \nabla \mathrm{G}_{1}\left(X_{k}\right)-\mathrm{G}_{1}\left(X_{k}\right) \nabla \mathrm{G}_{1}\left(X_{k}\right)}_{\text {local dynamics }}-\underbrace{\mathrm{c}_{k} \frac{\partial \mathrm{V}}{\partial X_{k}}(X)}_{\text {coupling dynamics }} \\
& \dot{\lambda}_{k, l, s}=\underbrace{-\mathrm{s}_{k, l, s}\left\langle\left(\begin{array}{c}
\frac{\partial \mathrm{V}}{\partial x_{k, l}}(X) \\
\frac{\partial \mathrm{V}}{\partial x_{k, s}}(X)
\end{array}\right) \mid\left(\begin{array}{l}
-\frac{\partial \mathrm{G}}{\partial x_{s}}\left(X_{k}\right) \\
-\frac{\partial \mathrm{G}}{\partial x_{l}}\left(X_{k}\right)
\end{array}\right)\right\rangle}_{\text {parametric dynamics }} \quad \begin{array}{l}
k=1, \ldots, N \\
l, s=1, \ldots, p . \\
s>l
\end{array}
\end{aligned}
$$

Let us discuss the existence of a consensual state and the convergence towards it.

Existence of a consensual state. The existence of a consensual state for System (3.2) is guaranteed if initial conditions are in $\mathcal{M}$ (cf. (2.6)) and $\Lambda_{k}:=\Lambda_{\mathrm{c}}$ for all $k$ with $\Lambda_{\mathrm{c}}$ a given constant vector.

Convergence towards a consensual state. The convergence towards a consensual state as well as the explicit value of the coefficients of $\Lambda_{\mathrm{c}}$ are established by Proposition 3.4. 
Proposition 3.4 Suppose that, for all $s$ and $l(l, s=1 \ldots, p$, and $s>l)$,

$$
\sum_{k=1}^{N}\left\langle\left(\begin{array}{c}
\frac{\partial \mathrm{V}}{\partial x_{k, l}}(X) \\
\frac{\partial \mathrm{V}}{\partial x_{k, s}}(X)
\end{array}\right) \mid\left(\begin{array}{c}
-\frac{\partial \mathrm{G}_{1}}{\partial x_{s}}\left(X_{k}\right) \\
-\frac{\partial \mathrm{G}_{1}}{\partial x_{l}}\left(X_{k}\right)
\end{array}\right)\right\rangle=0 \quad \forall X
$$

Then there exists a set $\mathcal{U} \supset \mathcal{C}_{\Lambda_{\mathrm{c}}}$ such that all orbits solving System (3.1) with initial conditions in $\mathcal{U}$ converge towards $\mathcal{C}_{\Lambda_{\mathrm{c}}}$ with $\mathcal{C}_{\Lambda_{\mathrm{c}}}:=\left\{(X, \Lambda) \in \mathbb{R}^{N p} \times \mathbb{R}^{N(p(p-1) / 2)} \mid X \in \mathcal{M}\right.$ and $\left.\Lambda=\mathbf{1} \otimes \Lambda_{\mathrm{c}}\right\}$ and the coefficients of $\Lambda_{\mathrm{c}}$ are

$$
\lambda_{c, l, s}:=\frac{\sum_{k=1}^{N}\left(\lambda_{k, l, s}(0) / s_{k, l, s}\right)}{\sum_{k=1}^{N}\left(1 / s_{k, l, s}\right)} \quad l, s=1 \ldots, p, \quad s>l,
$$

where 1 is a $p(p-1) / 2$-dimensional vector of 1 and $\otimes$ is the Kronecker product.

Proof. We refer the reader to Appendix F for the proof.

EXAMPLE 3.5 Let $p \geqslant 2$ be an arbitrary positive integer and the attracting submanifold be a hyperellipsoid in $\mathbb{R}^{p}$ of dimension $m=p-1$, defined by

$$
\mathrm{G}_{1}\left(X_{k}\right):=\sum_{j=1}^{p} \mathrm{a}_{j} x_{k, j}^{2}-1
$$

with $X_{k}=\left(x_{k, 1}, \ldots, x_{k, p}\right)$ and the $a_{j}$ are geometric parameters. The orthogonal part of the local dynamics is given by

$$
\mathrm{D}\left(X_{k}, \Lambda_{k}\right):=T_{k} \nabla \mathrm{G}_{1}\left(X_{k}\right)
$$

where the elements of $\Lambda_{k} \in \mathbb{R}^{p(p-1) / 2}$ define the $p \times p$ anti-symmetric matrix $T_{k}$. The coupling dynamics here is given by the Laplacian potential (we refer the reader to Example 2.6)

$$
\mathrm{V}(X):=\frac{1}{2} \sum_{j=1}^{p} \mathrm{a}_{j}\left\langle x_{j} \mid L x_{j}\right\rangle
$$

with $x_{j}:=\left(x_{1, j}, \ldots, x_{N, j}\right) \in \mathbb{R}^{N}$. The condition of Proposition 3.4 is satisfied because, for all $s$ and $l$ $(l, s=1 \ldots, p$, and $s>l)$,

$$
\sum_{k=1}^{N}\left\langle\left(\begin{array}{c}
\frac{\partial \mathrm{V}}{\partial x_{k, l}}(X) \\
\frac{\partial \mathrm{V}}{\partial x_{k, s}}(X)
\end{array}\right) \mid\left(\begin{array}{c}
-\frac{\partial \mathrm{G}}{\partial x_{s}}\left(X_{k}\right) \\
-\frac{\partial \mathrm{G}}{\partial x_{l}}\left(X_{k}\right)
\end{array}\right)\right\rangle=\sum_{k=1}^{N} \sum_{j=1}^{N} l_{k, j}\left(\mathbf{a}_{l} x_{j, l} \mathbf{a}_{s} x_{k, s}-\mathbf{a}_{s} x_{j, s} \mathbf{a}_{l} x_{k, l}\right)=0
$$


since, by Lemma B.1 in Appendix B, the last equality is zero. Note that, for $p \geqslant 3$, the orbit's geometry of the consensual state is not fully characterized: we only know that the orthogonal part of the local dynamics (i.e. D) has its orbits lying on the hyper-ellipsoid.

\subsection{Network of heterogeneous local dynamics with single-and multi-adapting flow parameters}

We now consider a network composed of both single- and multi-adapting parameters, as presented in Sections 3.2 and 3.3, respectively. For a fixed integer $0<v<N$, define the local dynamics as

$$
\begin{aligned}
& \text { for } k=1, \ldots, v: \mathrm{L}_{k}\left(X_{k}, \Lambda_{k}\right):=\lambda_{k} \mathrm{~K}\left(X_{k}\right)-\sum_{j \in I_{k}} \mathrm{G}_{j}\left(X_{k}\right) \nabla \mathrm{G}_{j}\left(X_{k}\right), \\
& \text { for } k=v+1, \ldots, N: \mathrm{L}_{k}\left(X_{k}, \Lambda_{k}\right):=T_{k} \nabla \mathrm{G}_{1}\left(X_{k}\right)-\mathrm{G}_{1}\left(X_{k}\right) \nabla \mathrm{G}_{1}\left(X_{k}\right),
\end{aligned}
$$

where the f-PV are $\Lambda_{k}=\lambda_{k}$ for $k=1, \ldots, v$ and $\Lambda_{k}=\Phi_{k} \in \mathbb{R}^{p(p-1) / 2}$ for $k=v+1, \ldots, N$, which defines a $p \times p$ anti-symmetric matrix $T_{k}$. If all the elements in $\Phi_{k}$ are equal to $\lambda_{\mathrm{c}}$, then $T_{k}=\lambda_{\mathrm{c}} T_{(\mathbb{1})}$ for all $k$, where $T_{(\mathbb{1})}$ is an anti-symmetric matrix with 1 on its upper diagonal. The dynamical system is

$$
\begin{aligned}
& \dot{X}_{k}=\underbrace{\mathrm{L}_{k}\left(X_{k} ; \Lambda_{k}\right)}_{\text {local dynamics }}-\underbrace{\mathrm{c}_{k} \frac{\partial \mathrm{V}}{\partial X_{k}}(X)}_{\text {coupling dynamics }} \quad k=1, \ldots, N \text {, } \\
& \dot{\lambda}_{k}=-\mathrm{s}_{k}\left\langle\frac{\partial \mathrm{V}}{\partial X_{k}}(X) \mid \mathrm{K}\left(X_{k}\right)\right\rangle \quad k=1, \ldots, v,
\end{aligned}
$$

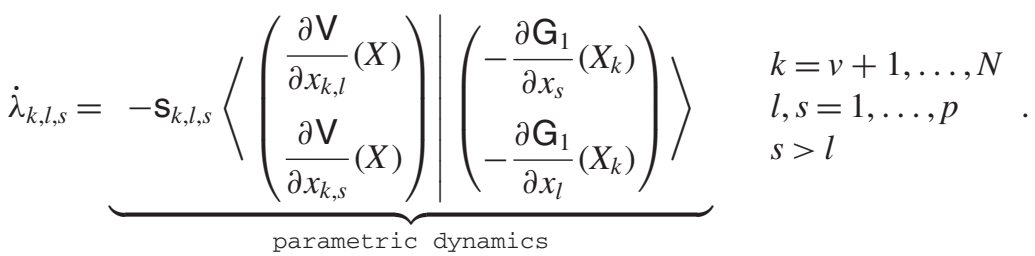

Let us discuss the existence of a consensual state and the convergence towards it.

Existence of a consensual state. The existence of a consensual state for System (3.3) is guaranteed if initial conditions are in $\mathcal{M}$ (cf. (2.6)) and we suppose that $\lambda_{\mathrm{c}} T_{(\mathbb{1})} \nabla \mathrm{G}_{1} \equiv \lambda_{\mathrm{c}} \mathrm{K}$ with $\lambda_{\mathrm{c}}$ a given constant.

Convergence towards a consensual state. The convergence towards a consensual state as well as the explicit value of $\lambda_{\mathrm{c}}$ and all coefficients of $\Lambda_{\mathrm{c}}$ are established by Proposition 3.6.

Proposition 3.6 Suppose that

$$
\sum_{k=1}^{v}\left\langle\frac{\partial \mathrm{V}}{\partial X_{k}}(X) \mid \mathrm{K}\left(X_{k}\right)\right\rangle+\sum_{\substack{s, l=1 \\
s<l}}^{p} \sum_{k=v+1}^{N}\left\langle\left(\begin{array}{c}
\frac{\partial \mathrm{V}}{\partial x_{k, l}}(X) \\
\frac{\partial \mathrm{V}}{\partial x_{k, s}}(X)
\end{array}\right) \mid\left(\begin{array}{c}
-\frac{\partial \mathrm{G}_{1}}{\partial x_{s}}\left(X_{k}\right) \\
-\frac{\partial \mathrm{G}_{1}}{\partial x_{l}}\left(X_{k}\right)
\end{array}\right)\right\rangle=0 \quad \forall X
$$

Then there exists a set $\mathcal{U} \supset \mathcal{C}_{\lambda_{\mathrm{c}} \times \Lambda_{\mathrm{c}}}$ such that all orbits solving System (3.1) with initial conditions in $\mathcal{U}$ converge towards $\mathcal{C}_{\lambda_{\mathrm{c}} \times \Lambda_{\mathrm{c}}}$ with $\mathcal{C}_{\lambda_{\mathrm{c}} \times \Lambda_{\mathrm{c}}}:=\left\{(X, \Lambda) \in \mathbb{R}^{N p} \times \mathbb{R}^{v+(N-v)(p(p-1) / 2)} \mid X \in \mathcal{M}\right.$ and $\left.\Lambda=\lambda_{\mathrm{c}} \mathbf{1}\right\}$ and 
$\lambda_{\mathrm{c}}$ and all coefficients of $\Lambda_{\mathrm{c}}$ are

$$
\lambda_{\mathrm{c}}:=\frac{\sum_{k=1}^{v}\left(\lambda_{k}(0) / \mathbf{s}_{k}\right)+\sum_{\substack{s, l=1 \\ s<l}}^{p} \sum_{k=v+1}^{N}\left(\lambda_{k, l, s}(0) / \mathbf{s}_{k, l, s}\right)}{\sum_{k=1}^{v}\left(1 / \mathbf{s}_{k}\right)+\sum_{\substack{s, l=1 \\ s<l}}^{p} \sum_{k=v+1}^{N}\left(1 / \mathbf{s}_{k, l, s}\right)},
$$

where 1 is a $v+(N-v)(p(p-1) / 2)$-dimensional vector of 1 .

Proof. We refer the reader to Appendix F for the proof.

ExAmple 3.7 Take $p=3$. For $k=1, \ldots, v$, define the local dynamics as the local dynamics in Example (3.3) with here $\mathrm{a}=\mathrm{b}=\mathrm{d}=1$ and with $\mathrm{G}_{2}(x, y, z):=\left(x-\frac{2}{3}\right)^{2}+\left(y-\frac{2}{3}\right)^{2}+\left(z-\frac{2}{3}\right)^{2}-$ 1 and gradient $\nabla \mathrm{G}_{2}(x, y, z)=2\left(x-\frac{2}{3}, y-\frac{2}{3}, z-\frac{2}{3}\right)$. For $k=v+1, \ldots, N$, define the local dynamics as the local dynamics in Example (3.5) with here $\mathrm{a}_{j}=1, j=1,2,3$. The coupling dynamics is as in Example (3.3) (with here $a=b=d=1$ ).

For $k=1, \ldots, v$, the resulting dynamical system is

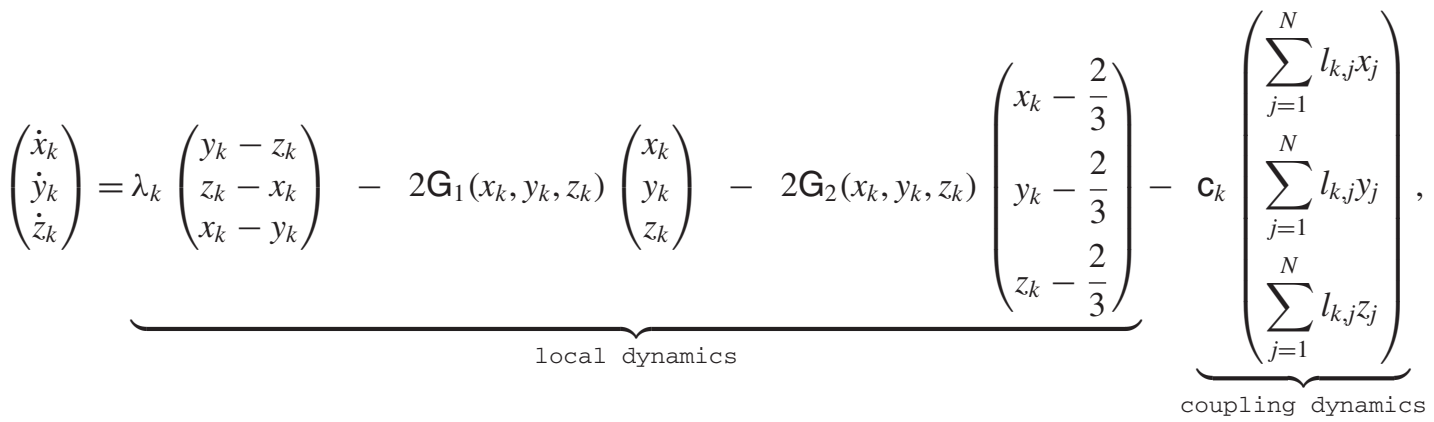

$$
\begin{aligned}
& \dot{\lambda}_{k}=\underbrace{-\mathrm{s}_{k}\left(\sum_{j=1}^{N} l_{k, j} x_{j}\left(y_{k}-z_{k}\right)+\sum_{j=1}^{N} l_{k, j} y_{j}\left(z_{k}-x_{k}\right)+\sum_{j=1}^{N} l_{k, j} z_{j}\left(x_{k}-y_{k}\right)\right)} . \\
& \text { parametric dynamics }
\end{aligned}
$$

For $k=v+1, \ldots, N$, the resulting dynamical system is

$$
\begin{aligned}
& \left(\begin{array}{l}
\dot{x}_{k} \\
\dot{y}_{k} \\
\dot{z}_{k}
\end{array}\right)=\underbrace{\left(\begin{array}{ccc}
0 & \lambda_{k, 1,2} & -\lambda_{k, 1,3} \\
-\lambda_{k, 1,2} & 0 & \lambda_{k, 2,3} \\
\lambda_{k, 1,3} & -\lambda_{k, 2,3} & 0
\end{array}\right)\left(\begin{array}{l}
x_{k} \\
y_{k} \\
z_{k}
\end{array}\right)-2 \mathrm{G}_{1}\left(X_{k}\right)\left(\begin{array}{l}
x_{k} \\
y_{k} \\
z_{k}
\end{array}\right)}_{\text {local dynamics }} \\
& \dot{\lambda}_{k, 1,2}=-\mathrm{s}_{k, 1,2}\left(\sum_{j=1}^{N} l_{k, j} x_{j} y_{k}-\sum_{j=1}^{N} l_{k, j} y_{j} x_{k}\right),
\end{aligned}
$$

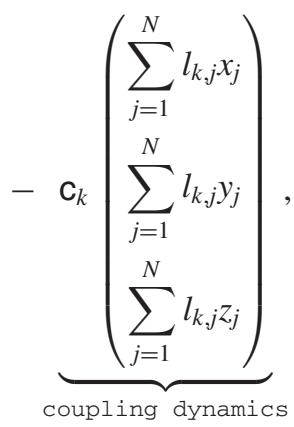




$$
\begin{aligned}
& \dot{\lambda}_{k, 1,3}=\mathrm{s}_{k, 1,3}\left(\sum_{j=1}^{N} l_{k, j} z_{j} x_{k}-\sum_{j=1}^{N} l_{k, j} x_{j} z_{k}\right) \\
& \dot{\lambda}_{k, 2,3}=\underbrace{-\mathbf{s}_{k, 2,3}\left(\sum_{j=1}^{N} l_{k, j} y_{j} z_{k}-\sum_{j=1}^{N} l_{k, j} z_{j} y_{k}\right)}_{\text {parametric dynamics }} .
\end{aligned}
$$

\section{Applications}

One field to which our results can readily be applied is 'soft control' (cf. Han et al. (2006)). The basic idea is to control the collective behaviour of the network while keeping the agents' local rule. This is done by introducing a 'shill' into the network. A 'shill' is an agent that is perceived by the whole community as an ordinary agent, but whose characteristics can be externally controlled. Similarly to an ordinary agent, a shill has —in general-limited power (i.e. it is not usually connected to all local systems). It interacts according to the same rules as any other local system. However, its local behaviour may be controlled externally and thus the collective dynamics may be influenced—or softly controlled—from the outside.

In our dynamical systems, a 'shill' may be perceived in two different ways.

(1) By determining the number of $\mathrm{G}_{j}$ functions in the local potential $\mathrm{E}_{k}$, one can control the shape of $\mathcal{L}$ (cf. 2.5) and hence where all local systems will converge. Consider Example 3.7 where here local dynamics do not have function $\mathrm{G}_{2}$ (for $k=1, \ldots, v$ ). In this case, the network will synchronize and all local systems will converge towards $\mathbb{S}^{2}$, but the orbit's geometry on the local attractor is not fully characterized. However, if the first system (i.e. $k=1$ ) is equipped with functions $\mathrm{G}_{2}$, then, asymptotically, the whole network evolves on a particular circle of $\mathbb{S}^{2}$ (the intersection of the two spheres in Fig. 1). Hence, regarding the consensual manifold, local dynamics $k=1$ is a shill and softly controls the global dynamical system.

(2) By determining the values of the susceptibility constants, one can control the consensual values of the f-PV. Indeed, all constants of motion presented in this contribution are of the form $\mathrm{J}\left(\lambda_{1}, \ldots, \lambda_{u}\right)=\sum_{k=1}^{u}\left(\lambda_{k} / \mathbf{s}_{k}\right)$ with $\mathbf{s}_{k}>0$ and hence the respective consensual value reads

$$
\lambda_{\mathrm{c}}=\frac{\sum_{k=1}^{u}\left(\lambda_{k}(0) / \mathrm{s}_{k}\right)}{\sum_{k=1}^{u}\left(1 / \mathbf{s}_{k}\right)},
$$

where $u$ is equal to $N$ for all constants of motion in Sections 3.2 and 3.3 and equal to $v+(N-v)(p(p-1) / 2)$ in Section 3.4. Consensual values for the local parameters are hence weighted averages and therefore local systems with small-valued susceptibility constants (i.e. 'stubborn' agents) have more influence on the resulting consensual values. Hence, concerning the consensual values, a 'stubborn' local dynamics is a shill and softly controls the asymptotic values of the f-PV.

An actual realization of a shill is given in Faria et al. (2010), where a 'robot fish' is introduced into a tank with other living fish of the same species. The 'robot fish' is controlled by the experimenter and thus different types of interactions (e.g. leading the collection of fish into a certain region) can be thoroughly studied. In our case, we may introduce a shill robot (controllable robot) into a swarm 
of autonomous interacting robots. This way, one would be able to softly control the consensual state emerging from self-organizing entities.

This type of control arises in robot formation modelling, a research field that tackles the problem of making a robot community converge towards a specific curve in space. Once the required formation is attained, it has to be maintained as long as necessary. Collective evolutions of robot teams are presently receiving attention for a wide range of applications. For example, platoons of robots may be used for (1) travelling over areas of high risks of floods or natural fires for close observation and data gathering, (2) exploring new environments such as caves or ocean floors or (3) patrolling around minefields or cordoning the perimeter of accidents or environmental catastrophes.

Specifically, such a type of robot dynamics is explicitly considered in Hsieh et al. (2007) and it exhibits close similarities with our dynamics. The authors study the following dynamical system

$$
\dot{X}_{k}=-\mathrm{R}(X) \nabla \mathrm{E}\left(X_{k}\right)+\mathrm{S}(X) \mathrm{D}\left(X_{k}\right),
$$

where $X_{k}=\left(x_{k}, y_{k}\right)$ denotes the position of the $k$ th robot, $X:=\left(X_{1}, \ldots, X_{N}\right)$, and $\mathrm{E}$ and $\mathrm{D}$ form an $\mathrm{O}-\mathrm{G}$ system. The authors call the positive semi-definite function $\mathrm{E}$ the shape navigation function. The gradient $\nabla E$ informs each robot where to go, and the vector $D$ describes how each robot must circulate on the specific curve. The positive scalar functions $\mathrm{R}$ and $\mathrm{S}$ modulate the gradient and orthogonal parts of the local systems in order to avoid collisions. Since these two functions are to be seen as coupling dynamics, the system is of a multiplicative coupling nature.

In Hsieh et al. (2007), robots are assumed to be programmed with identical behaviour(i.e. homogeneous local dynamics with identical valued parameters). However, because of the omnipresence of noise in nature, it is realistic to presume that every robot receives the same $D$, but with different valued flow parameters. For small mismatches in the local flow parameters, the overall effect of the dynamics is not significantly modified: robots do converge without collisions towards a specific curve. However, the mismatches impose that local systems must constantly communicate with their neighbours to maintain the dynamical pattern. The robots have different information regarding their flow parameters and thus a steady amount of information is mandatory to regulate all individual systems.

To maintain a robot formation with as little communication and human supervision as possible (as stated in Hsieh et al., 2007), a potential solution would be to rely on parametric adaptive mechanisms that enable the robots to minimize central coupling (i.e. be less dependent on communications).

\section{Numerical illustrations}

We first display numerical simulations of two-dimensional O-G systems to clearly present how the consensual value $\lambda_{c}$ is influenced by the constant of motion (we refer the reader to Section 5.1). We then show the adaptive mechanisms at work in networks of three-dimensional O-G systems (refer to Section 5.2). In addition, we numerically show that the orthogonality property on the adaptive mechanism (implying Equation (2.14)) can be relaxed and the oscillators still adapt to reach a common angular velocity (refer to Section 5.3).

\subsection{Two-dimensional $O-G$ systems}

Consider a network of five local dynamics as in Equations (1.4) (i.e. Hopf oscillators) with two different topologies: 'Crystal' (cf. Fig. 4(c)) and 'All-to-One' (cf. Fig. 4(d)). The coupling strengths are chosen as $\mathrm{C}_{k}=1$ for all $k$ and the susceptibility constants as $\mathrm{s}_{1}=4, \mathrm{~s}_{2}=\frac{5}{4}, \mathrm{~s}_{3}=0.1, \mathrm{~s}_{4}=\frac{4}{3}, \mathrm{~s}_{5}=5$. 
(a)

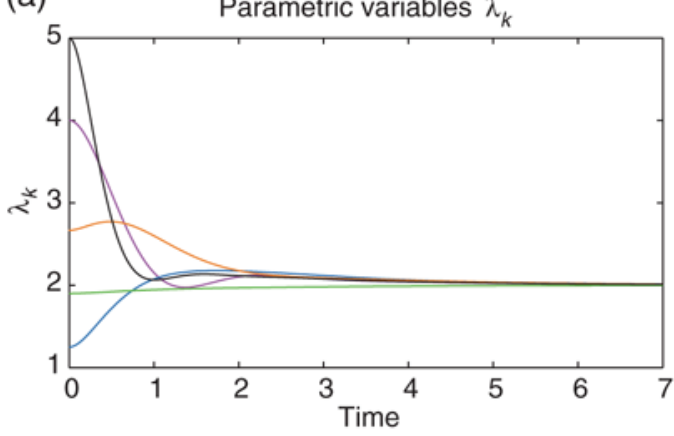

(c)

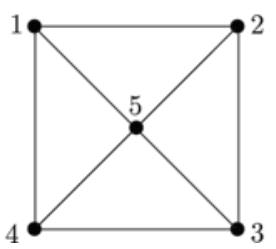

(b)

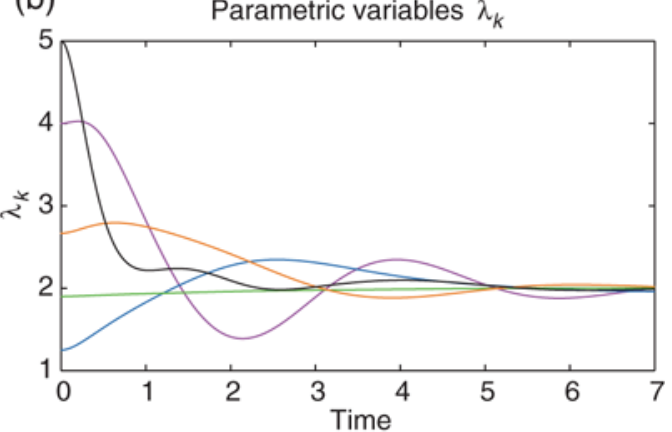

(d)

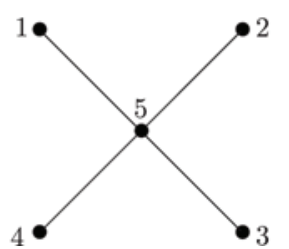

FIG. 4. Time evolution of the parametric variables $\lambda_{k}(\mathrm{a}$ and b) for five Hopf oscillators interacting through a 'Crystal' network (c) $(\mathrm{AC}=3)$ and an 'All-to-One' network $(\mathrm{d})(\mathrm{AC}=1)$, respectively.

The initial conditions for the state variables are $x_{k}(0)=1$ and $y_{k}(0)=0$ for all $k$ and for the parametric variables $\lambda_{1}(0)=4, \lambda_{2}(0)=\frac{5}{4}, \lambda_{3}(0)=1.9, \lambda_{4}(0)=\frac{8}{3}, \lambda_{5}(0)=5$ (i.e. they are identical to their susceptibility constants except for $k=3$ and $k=4$ ). The transient dynamics of the adaptive mechanism is shown in Fig. 4.

In Fig. 4(a,b), the green trajectory (corresponding to vertex 3) barley changes values, while all other trajectories converge towards it. The local system at vertex 3 has a susceptibility constant close to zero and therefore acts as a shill to softly control the asymptotic values of the f-PV. Indeed, computing the constant of motion with the given values, one obtains

$$
\lambda_{\mathrm{c}}=\frac{\sum_{k=1}^{5} \lambda_{k}(0) / \mathrm{s}_{k}}{\sum_{k=1}^{5} 1 / \mathrm{s}_{k}}=\frac{5+\lambda_{3}(0) 10}{12}=2
$$

with $\lambda_{3}(0)=1.9$. Both Fig. 4(a,b) have the same time scale. This shows that the convergence rate manifestly depends on the algebraic connectivity (AC): the larger the AC, the faster the convergence. Linearization around the consensual state explicitly shows the interplay between the AC and the convergence rate (see Rodriguez \& Hongler, 2009a,b). Thus, the convergence rate explicitly depends on the topology of the network, but not on the consensual value $\lambda_{\mathrm{c}}$.

\subsection{Three-dimensional $O-G$ systems}

We perform numerical simulations with 30 homogeneous local dynamics (we refer the reader to Section 5.2.1) and with 10 heterogeneous local dynamics (we refer the reader to Section 5.2.2). For each case, three different types of network topology are considered: (i) 'Randomly Distributed' 


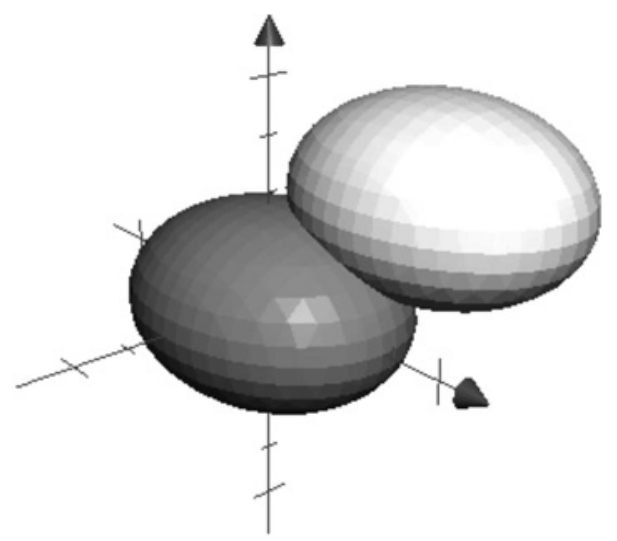

FIG. 5. Two ellipsoids $\mathrm{G}_{1}(X):=2 x^{2}+3 y^{2}+5 z^{2}-1$ (dark gray) and $\mathrm{G}_{2}(X):=2\left(x-\frac{1}{2}\right)^{2}+3\left(y-\frac{1}{2}\right)^{2}+5\left(z-\frac{1}{2}\right)^{2}-1$ (light gray).
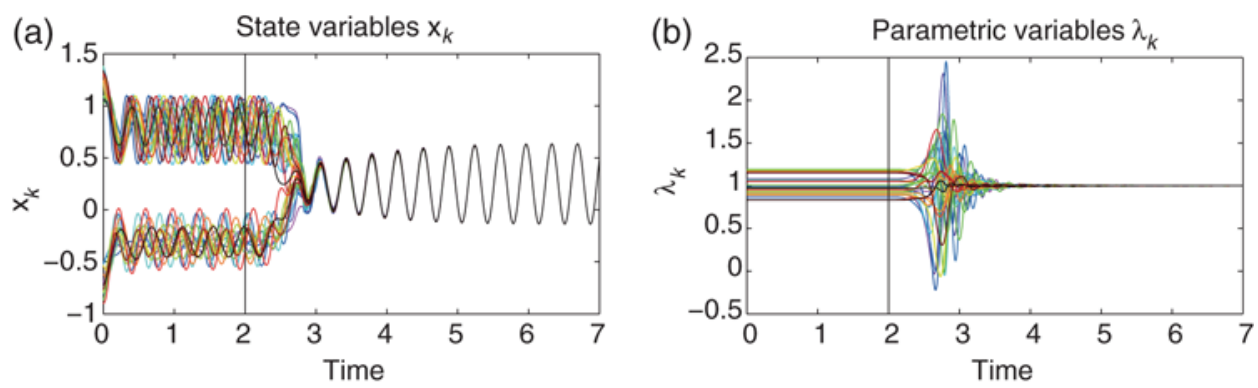

FIG. 6. Time evolution of the state variables $x_{k}$ (a) and parametric variables $\lambda_{k}$ (b) for 30 homogeneous 1 ocal dynamics with ellipsoidal attractor as in Fig. 5 interacting through a 'Randomly Distributed' network (AC=4.6049). Coupling dynamics and parametric dynamics are switched on at $t=2$ (black solid line).

networks, (ii) 'All-to-All' networks and (iii) 'All-to-One' networks (i.e. interactions are only through the $N$ th local dynamics). For the case (i), the edges $a_{l, s}$ (i.e the entries of the symmetric adjacency A) of the 'Randomly Distributed' network are determined as follows:

- the $N$ th node is connected to all other nodes (in order to guarantee that the network is connected) with intensity one: $a_{N, s}=1$ for $s=1, \ldots, N-1$;

- all other edges are the product of two random variables: $a_{l, s}=\mathrm{ZI}$ for $l, s=1, \ldots, N-1$ and $l<s$. $Z$ is a Bernoulli random variable taking 0 or 1 as a value with probability $\frac{1}{2}$, and $I$ is uniformly distributed on the interval $[0,1]$;

- no loops are allowed: $a_{l, l}=0$ for $l=1, \ldots, N$.

We choose the coupling strengths $\mathbf{C}_{k}$ and susceptibility constants $\mathbf{S}_{k}$ as

$$
\mathrm{c}_{k+1}:=\mathrm{c}_{1}+k \frac{0.75}{N-1} \quad \mathrm{~s}_{k+1}:=\mathrm{s}_{1}-k \frac{2.5}{N-1} \text { for } k=1, \ldots, N-1
$$

with $\mathrm{c}_{1}:=0.25$ and $\mathrm{s}_{1}:=3.5$ (and therefore $\mathrm{c}_{N}=\mathrm{s}_{N}=1$ ). For the network of heterogeneous local dynamics, we choose $\mathbf{s}_{k, 1,2}=\mathbf{s}_{k, 1,3}=\mathbf{s}_{k, 2,3}=\mathbf{s}_{k}$ for $k=v+1, \ldots, N$. 

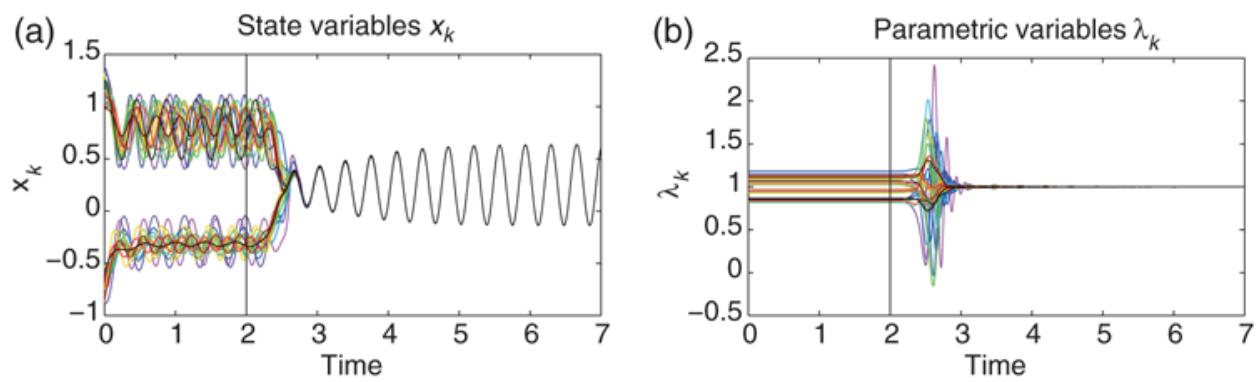

FIG. 7. Time evolution of the state variables $x_{k}$ (a) and parametric variables $\lambda_{k}$ (b) for 30 homogeneous local dynamics with ellipsoidal attractor as in Fig. 5 interacting through a 'All-to-All' network $(\mathrm{AC}=30)$. Coupling dynamics and parametric dynamics are switched on at $t=2$ (black solid line).

5.2.1 Homogeneous local dynamics. For the 30 homogeneous local dynamics, D is defined as in Example (3.3) and we choose $\mathrm{G}_{1}\left(X_{k}\right):=\mathrm{a} x_{k}^{2}+\mathrm{b} y_{k}^{2}+\mathrm{c} z_{k}^{2}-1$ and $\mathrm{G}_{2}\left(X_{k}\right):=\mathrm{a}\left(x_{k}-\frac{1}{2}\right)^{2}+\mathrm{b}\left(y_{k}-\right.$ $\left.\frac{1}{2}\right)^{2}+\mathrm{c}\left(z_{k}-\frac{1}{2}\right)^{2}-1$ with $X_{k}=\left(x_{k}, y_{k}, z_{k}\right)$ and $\mathrm{a}=2, \mathrm{~b}=3$ and $\mathrm{c}=5$. These are two identical ellipsoids, with $\mathrm{G}_{1}$ centred at the origin and $\mathrm{G}_{2}$ centred at $\left(\frac{1}{2}, \frac{1}{2}, \frac{1}{2}\right)$, as shown in Fig. 5. The potentials are defined as: $\mathrm{E}_{k} \equiv \frac{1}{2} \mathrm{G}_{1}^{2}$ if $k$ is odd and $\mathrm{E}_{k} \equiv \frac{1}{2} \mathrm{G}_{2}^{2}$ if $k$ is even. For each network topology, initial conditions $x_{k}(0)$ are randomly uniformly distributed on the following intervals: $[-1 / \sqrt{\mathrm{a}}-0.2,-1 / \sqrt{\mathrm{a}}+0.2]$ if $k$ is odd and on $\left[\frac{1}{2}+1 / \sqrt{\mathbf{a}}-0.2, \frac{1}{2}+1 / \sqrt{\mathbf{a}}+0.2\right]$ if $k$ is even. The same applies for $y_{k}(0)$ and $z_{k}(0)$ with, respectively, $\mathrm{b}$ and $\mathrm{c}$ instead of $\mathrm{a}$. The initial conditions $\lambda_{k}(0)$ of the $\mathrm{f}-\mathrm{PV}$ are randomly (uniform distribution) drawn from the interval $[0.8,1.2]$ and are rescaled so that the constant of motion is one.

Figures 6-8 show, respectively, the resulting dynamics for the state variables $x_{k}$ and the adaptive mechanism (i.e. parametric variables $\lambda_{k}$ ) with the three types of network: 'Randomly Distributed', 'Allto-All' and 'All-to-One'. The AC for each network is reported. To better perceive the convergence, Fig. 8 runs on a longer time scale.

For $t \in[0,2]$, the coupling dynamics and the parametric dynamics are switched off (i.e. $\mathrm{C}_{k} \equiv \mathrm{P}_{k} \equiv 0$ for all $k$ ) -local dynamics are governed by their local parameters and attractors. At $t=2$, interactions are switched on (see black solid line). Switching on is done with a smooth function that changes from 0 to 1 on a unit time interval (see Appendix B in Rodriguez \& Hongler (2009a) for details). In all simulations, one can observe that during the decoupled phase (i.e. for $t \in[0,2])$ local systems converge towards their attractor (either one of the ellipsoids in Fig. 5). Once coupling dynamics and parametric dynamics are switched on, all local systems converge towards the common attractor, here the intersection of the two ellipsoids.

5.2.2 Heterogeneous local dynamics. For the 10 local dynamics defined in Example (3.7), we choose $v=3$. For each network topology, initial conditions $\left(x_{k}(0), y_{k}(0), z_{k}(0)\right)$ are randomly uniformly distributed on $[-0.25,0.25]^{3}$ for $k=1, \ldots, N$. The initial conditions of the $\mathrm{f}-\mathrm{PV}$ are randomly (uniform distribution) drawn from the interval $[-1,1]$ for $\lambda_{k}(0)$ (for $\left.k=1,2,3\right)$ and from the intervals $[0.8,1.3]$ for $\alpha_{k}(0),[-1,1]$ for $\beta_{k}(0)$ and $[1.8,2.5]$ for $\lambda_{k, 2,3}(0)$ (for $\left.k=4, \ldots, 10\right)$. They are rescaled so that the constant of motion is 1 .

Figures 9-11 show, respectively, the resulting dynamics for the state variables $z_{k}$ and the adaptive mechanism (i.e. parametric variables $\lambda_{k}, \lambda_{k, 1,3}$ and $\lambda_{k, 2,3}$ ) with the three types of network: 'Randomly 

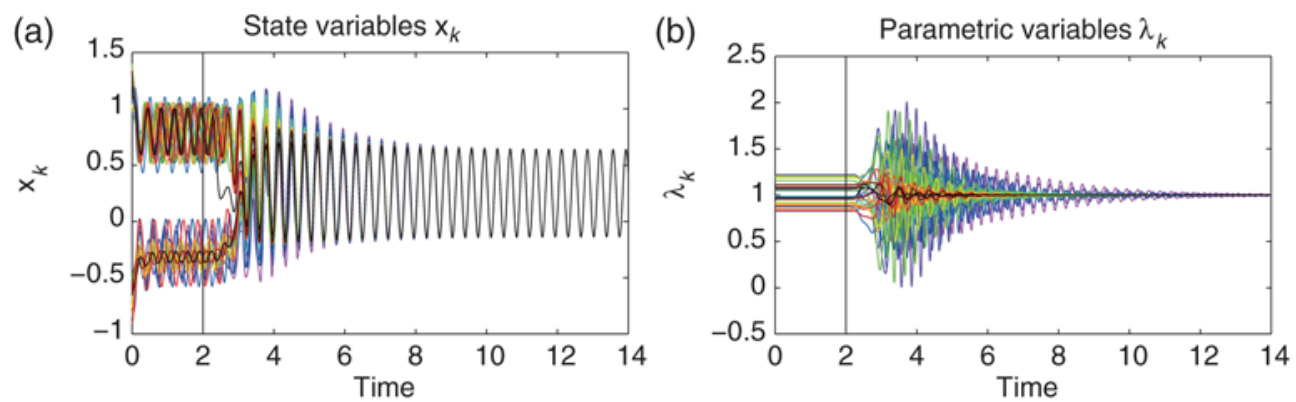

FIG. 8. Time evolution of the state variables $x_{k}$ (a) and parametric variables $\lambda_{k}$ (b) for 30 homogeneous 1 ocal dynamics with ellipsoidal attractor as in Fig. 5 interacting through a 'All-to-One' network $(\mathrm{AC}=1)$. Coupling dynamics and parametric dynamics are switched on at $t=2$ (black solid line).
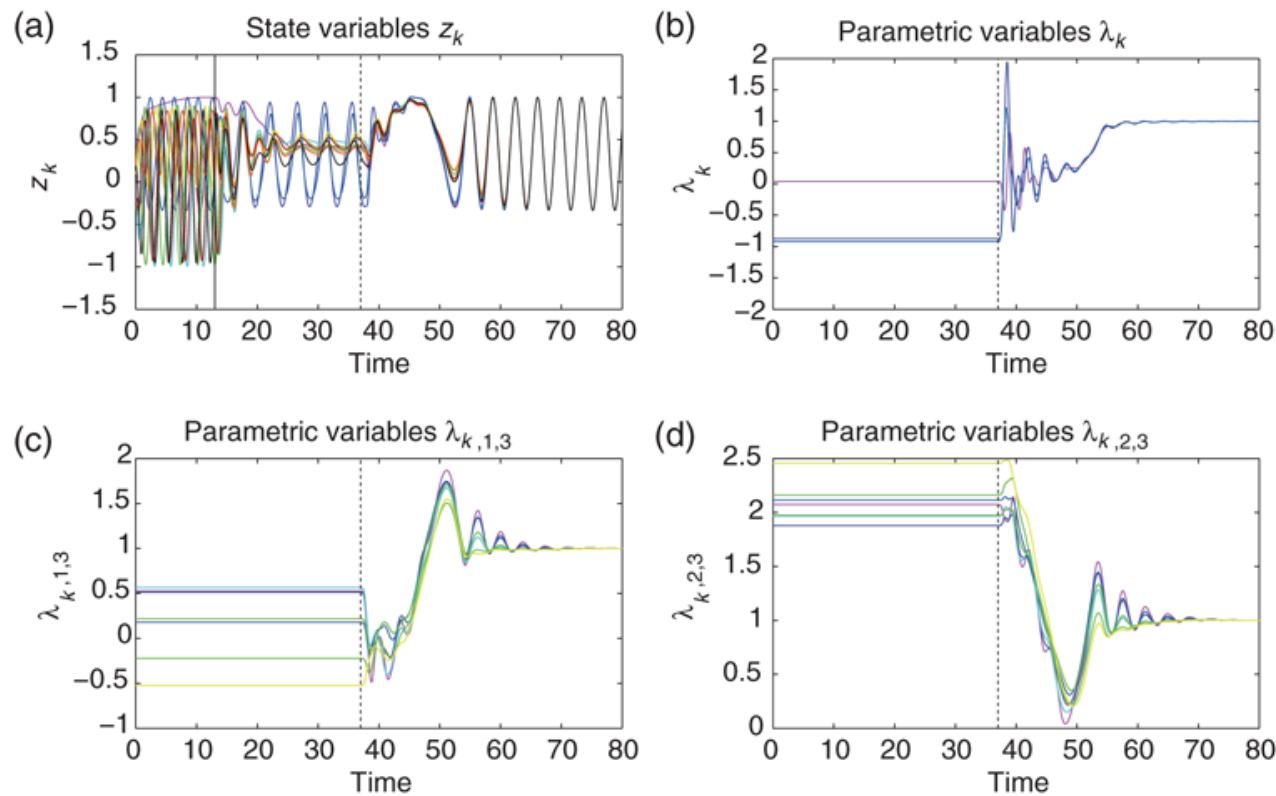

FIG. 9. Time evolution of the state variables $z_{k}$ (a) and parametric variables $\lambda_{k}, \lambda_{k, 1,3}$ and $\lambda_{k, 2,3}$ (b-d) for 10 heterogeneous local dynamics defined in Example (3.7) interacting through a 'Randomly Distributed' network (AC $=1.3825$ ). Coupling dynamics are switched on at $t=13$ (black solid line) and parametric dynamics at $t=37$ (black dashed line).

Distributed', 'All-to-All' and 'All-to-One'. The AC for each network is reported. To better perceive the convergence, Fig. 11 runs on a longer time scale.

For $t \in[0,13]$, neither the coupling dynamics nor the parametric dynamics are switched on (i.e. $\mathrm{C}_{k} \equiv \mathrm{P}_{k} \equiv 0$ for all $k$ )-local dynamics are governed by their local parameters and attractors. At $t=13$, only coupling dynamics are switched on (see the black solid line) and finally, at $t=37$, parametric dynamics are switched on (see the black dashed line). Note that, for the 'All-to-All' network, the 'ordered' state reached between $t=13$ and $t=37$ (i.e. when there is no adaptive mechanism in effect $-\mathrm{P}_{k} \equiv 0$ for all $k$ ) is a trivial dynamics (fixed point), whereas for 

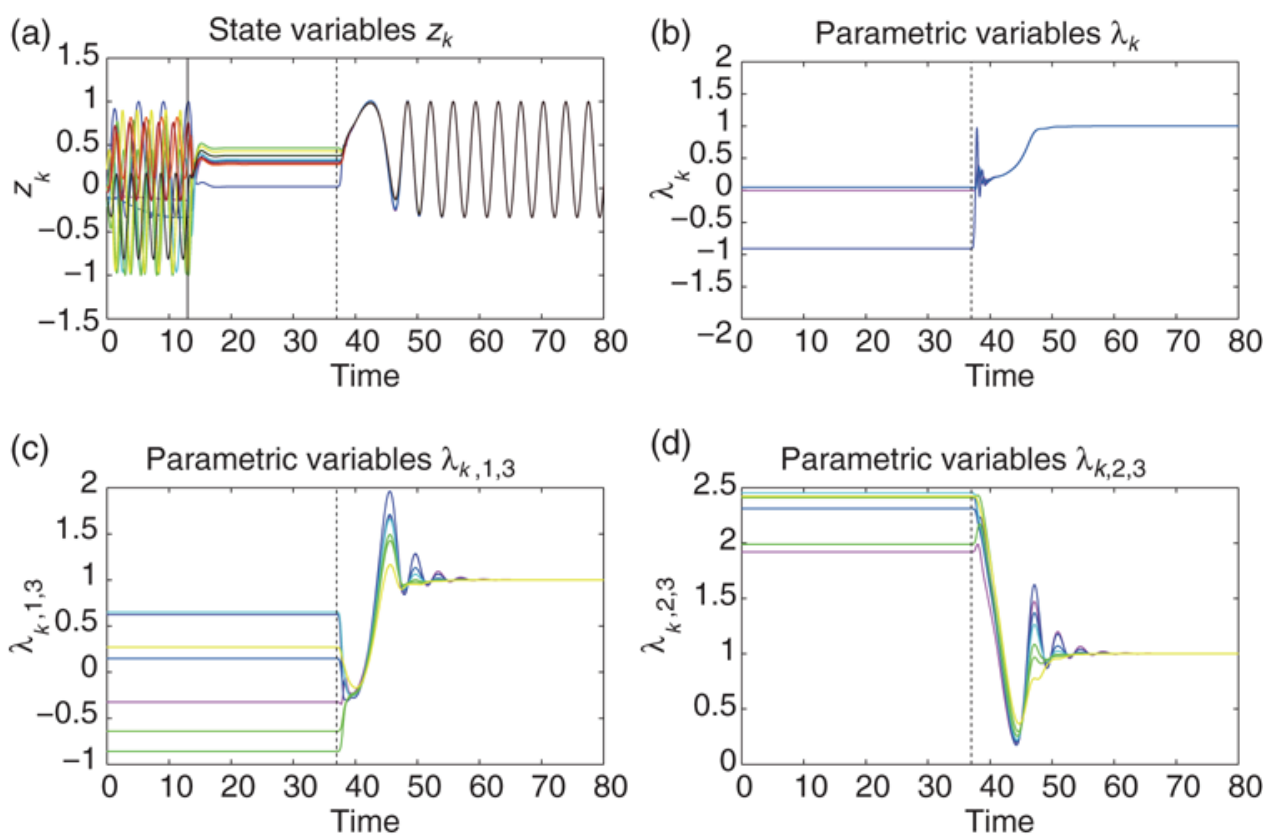

FIG. 10. Time evolution of the state variables $z_{k}$ (a) and parametric variables $\lambda_{k}, \lambda_{k, 1,3}$ and $\lambda_{k, 2,3}$ (b-d) for 10 heterogeneous local dynamics defined in Example (3.7) interacting through a 'All-to-All' network (AC = 10). Coupling dynamics are switched on at $t=13$ (black solid line) and parametric dynamics at $t=37$ (black dashed line).

(a)

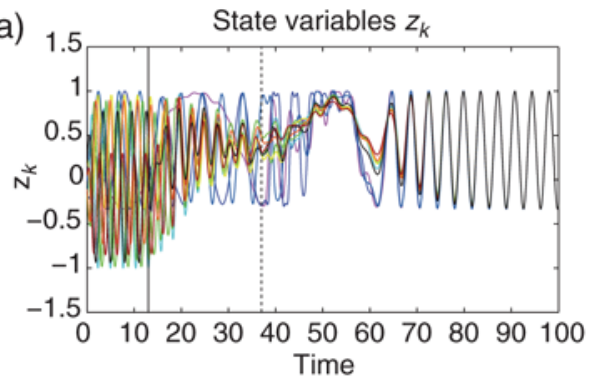

(c)

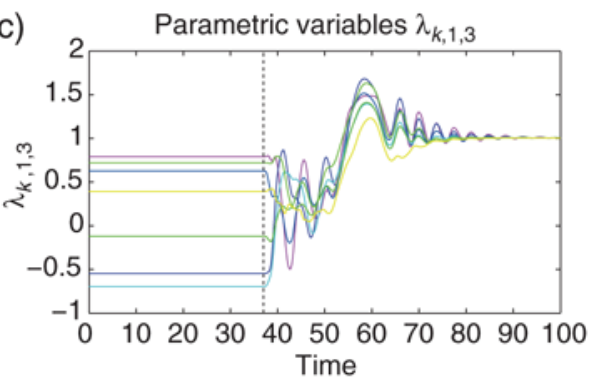

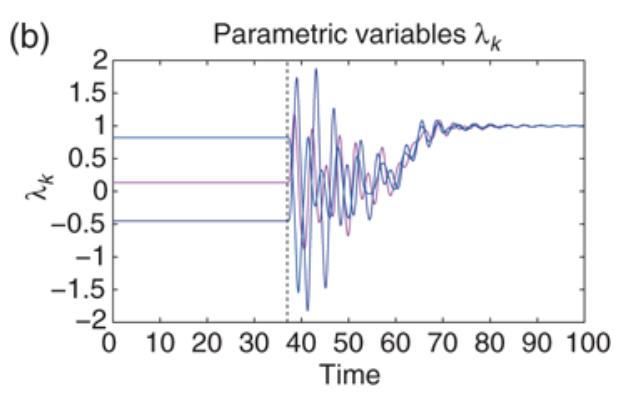

(d)

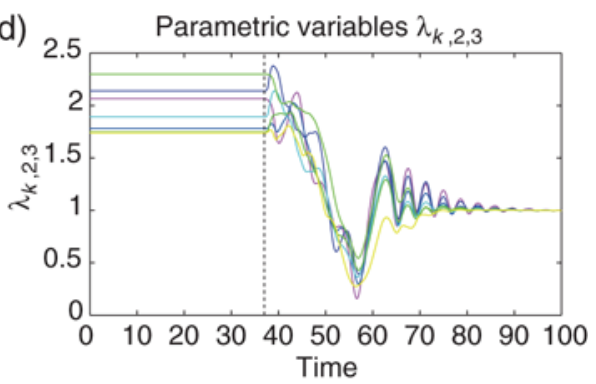

FIG. 11. Time evolution of the state variables $z_{k}$ (a) and parametric variables $\lambda_{k}, \lambda_{k, 1,3}$ and $\lambda_{k, 2,3}$ (b-d) for 10 heterogeneous local dynamics defined in Example (3.7) interacting through a 'All-to-One' network (AC = 1). Coupling dynamics are switched on at $t=13$ (black solid line) and parametric dynamics at $t=37$ (black dashed line). 
the 'Randomly Distributed' and 'All-to-One' network cyclo-stationary states seem to be attained. We observe this phenomenon in numerical experiments where the time interval on which the coupling dynamics act has been enlarged.

\subsection{Non-orthogonal adaptive mechanisms}

To investigate the network's dynamics when the hypothesis in Equation (2.12) is relaxed (i.e. nonorthogonal adaptive mechanism), we perform two numerical investigations, each having five limit cycle oscillators interacting through a 'Crystal' type network (cf. Fig. 4(c)). The first numerical experiment concerns Hopf oscillators (we refer the reader to Section 5.3.1), whereas the second one deals with Van der Pol oscillators (i.e. non-O-G systems) (we refer the reader to Section 5.3.2). In both cases, the initial conditions for the state variables $\left(x_{k}(0), y_{k}(0)\right)$ are randomly uniformly distributed on $[-1,1]^{2}$ and the initial conditions of the $\mathrm{f}-\mathrm{PV} \lambda_{k}(0)$ are randomly (uniform distribution) drawn from the interval $[0.5,1.5]$. These are rescaled so that the average value is 1 .

5.3.1 Hopf oscillators. The system is given as in Equations (1.4), with all coupling strengths and susceptibility constants set to 1 . Here, the parametric dynamics for the $\lambda_{k}$ is given by

$$
\dot{\lambda}_{k}=-\sum_{j=1}^{5} l_{k, j} x_{j} y_{k} \quad k=1, \ldots, 5 .
$$

Figure 12 displays the resulting dynamics for the state variables $y_{k}$ and the adaptive mechanism (i.e. parametric variables $\lambda_{k}$ ) with a 'Crystal' type network. For $t \in[0,15]$, neither the coupling dynamics nor the parametric dynamics are switched on. After the coupling and the adaptive mechanisms are switched on, all $\lambda_{k}$ converge towards a common and constant value which is not equal to 1-1 being the rescaled average value of the initial $\lambda_{k}$ distribution and the value of the consensual frequency $\lambda_{c}$ if Equation (2.12) is satisfied.

5.3.2 Van der Pol oscillators. The dynamical system of the network consists of five diffusively coupled Van der Pol oscillators (i.e. non-O-G systems). Each oscillator's parametric variable $\lambda_{k}$ multiplies the whole local vector field and thus controls the angular velocity on its limit cycle (i.e. $\lambda_{k}$ plays the
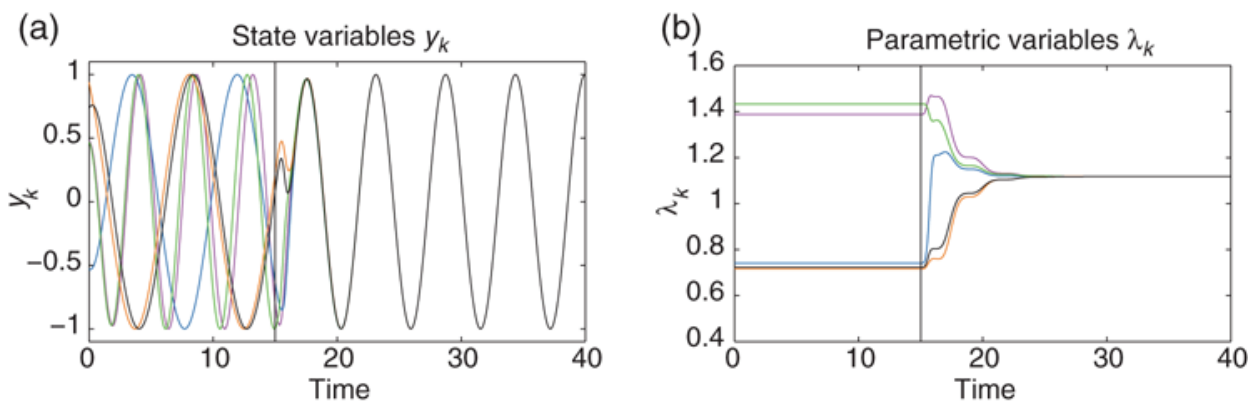

FIG. 12. Time evolution of the state variables $y_{k}$ (a) and parametric variables $\lambda_{k}$ (b) for five Hopf oscillators interacting through a 'Crystal' type network. Coupling dynamics and parametric dynamics are switched on at $t=15$ (black solid line). 
(a)

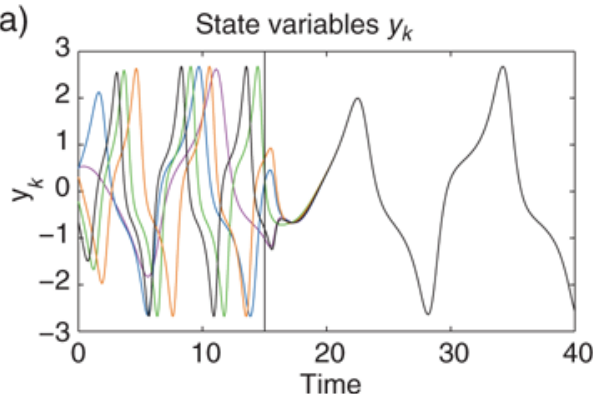

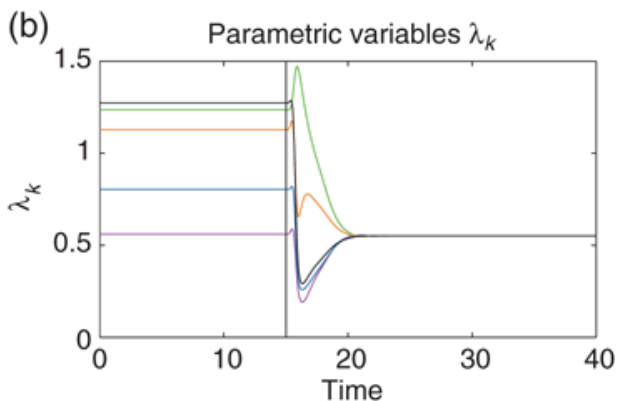

FIG. 13. Time evolution of the state variables $y_{k}$ (a) and parametric variables $\lambda_{k}$ (b) for five Hopf oscillators interacting through a 'Crystal' type network. Coupling dynamics and parametric dynamics are switched on at $t=15$ (black solid line).

role of a flow parameter). A parametric dynamics not satisfying the orthogonality property in the adaptive mechanism is introduced. The dynamical system is

$$
\begin{aligned}
& \dot{x}_{k}=\lambda_{k} y_{k}- \\
& \dot{y}_{k}=\underbrace{\lambda_{k}\left(\left(1-x_{k}^{2}\right) y_{k}-x_{k}\right)}_{\text {local dynamics }}-\underbrace{N}_{\text {coupling dynamics }} l_{k, j} x_{k} \\
& \dot{\lambda}_{k}=\underbrace{N}_{\text {parametric dynamics }} \underbrace{\sum_{j=1}^{N} l_{k, j} y_{k}\left(x_{j} y_{k}+y_{j}\left(\left(1-x_{k}^{2}\right) y_{k}-x_{k}\right)\right.}_{j=1})
\end{aligned}
$$

Figure 13 shows the resulting dynamics for the state variables $y_{k}$ and the adaptive mechanism (i.e. parametric variables $\lambda_{k}$ ) with a 'Crystal' type network. As in Section 5.3.1, the coupling dynamics and the parametric dynamics are switched on at $t=15$. Equally as in Section 5.3.1, observe that all $\lambda_{k}$ converge towards a common and constant value, which is, however, not equal to 1 .

\section{Conclusion and perspectives}

The interactions between agents enable synchronization and/or learning mechanisms from which collective dynamic patterns emerge. The synchronization mechanisms, first exposed in the seminal contribution of Christiaan Huygens, paved the way for a century-long and still ongoing multidisciplinary research. Recently, growing attention is being paid to dynamic learning and adaptive issues arising in networks of interacting dynamical systems. Synchronization and adaptation can be viewed as somehow complementary mechanisms. Indeed, while synchronization expresses an 'elastic capability' enabling dynamical systems to produce ephemeral common dynamical patterns, adaptive systems exhibit a 'plastic capability' enabling the formation of permanent common dynamical patterns. The word associations elastic-ephemeral versus plastic-permanence emphasize that classical synchronization patterns are produced owing to the steady action of mutual interactions-remove interactions and all local evolutions 
return to their original eigen-dynamics (i.e. chasser le naturel et il revient au galop) ${ }^{9}$ Conversely, adaptive dynamics permanently alter the local dynamics-even after interactions are removed, local evolutions do not return to their original eigen-dynamics.

To analytically discuss adaptation along the lines just exposed, we introduced a class of stylized models which are based on the following assumptions

(1) Local dynamics on each network vertex is characterized by O-G Systems. This ensures the existence of stable local attractors.

(2) Mutual interactions are derived from the gradient of a coupling potential. Hence, the underlying network is undirected.

(3) Orthogonality in the adaptive mechanism (i.e. implying Equation (2.14)) allows for the existence of constants of motion for the parametric variables, enabling the full determination of consensus.

The orthogonality property in the adaptive mechanism is rather restrictive. Numerical experiments, however, show that this property can be relaxed, and that a consensual angular velocity is still reached. On the other hand, determining the exact value of the angular velocity remains an open problem.

Among the vast range of situations leading to dynamical adaptation, we focused on a simple and idealized class for which permanent self-tuning of individual control parameters is analytically tractable. As usual, what is gained for the simplicity of solvable models has to be paid for by limiting the generality. We still think that the insights offered by our models do raise several relevant questions for future investigations.

While our models offer the possibility to explicitly calculate the consensual evolution, further analytical results for more general situations remain to be developed. In particular, ongoing research is devoted to consider more general interactions for which the network connectivity affects the consensual dynamics. Such interactions can be observed in the adaptive behaviour of societies of interacting agents like platoons of birds, schools of fish, gregarious grasshoppers, etc. Here the agents' density and their interconnectivity ultimately determines their dynamical behaviour (cf. Cucker \& Smale, 2007). Also, the restriction to time-independent networks has to be relaxed to cover other applications, involving non-stationary and noisy environments-which arise in the real world. Recent studies show how timedependent connectivity may either stabilize or destabilize the dynamics via parametric and/or stochastic resonance (cf. Rodriguez \& Hongler, 2010). The nature of the interactions together with the network connectivity could alternatively be studied in the context of optimal control theory, provided a set of relevant objective functions are defined. Finally, allowing a distinction between physical and informationdirected networks (i.e. breaking the symmetry of the adjacency matrix) opens new and unexplored questions for the resulting adaptation capabilities.

\section{Acknowledgements}

The authors thank Dr M. Anderegg for his inspirations derived from valuable discussions and suggestions, and his constructive criticisms and insightful comments that were very beneficial to this work. He directly collaborated in the results and proofs of Lemma E.1 and Corollary E.2.

\footnotetext{
${ }^{9}$ Drive out natural and personal behaviour and it will be back in full gallop.
} 


\section{Funding}

This research was partially supported by the Swiss National Science Foundation (200021-126502 to J.R.).

\section{REFERENCES}

ACEBRón, J. \& Spigler, R. (1998) Adaptive frequency model for phase-frequency synchronization in large populations of globally coupled nonlinear oscillators. Phys. Rev. Lett., 81, 2229-2232.

Bhatia, N. P. \& Szegö, G. P. (1970) Stability Theory of Dynamical Systems. Berlin: Springer.

Blekhman, I. I. (1988) Synchronization in Science and Technology. New York: ASME Press.

Boccaletti, S., Hwang, D.-U., Chavez, M., Amann, A., Kurths, J. \& Pecora, L. M. (2006) Synchronization in dynamical networks: evolution along commutative graphs. Phys. Rev. E, 74, 016102.

Buchli, J. \& IJSPEert, A. J. (2004) A simple, adaptive locomotion toy-system. Proceedings of Eighth International Conference on the Simulation of Adaptive Behavior (SAB'04). Cambridge, Massachusetts: MIT Press.

Chavez, M., Hwang, D.-U., Amann, A., Hentschel, H. \& Boccaletti, S. (2005) Synchronization is enhanced in weighted complex networks. Phys. Rev. Lett., 94, 218701.

Cucker, F. \& Smale, S. (2007) Emergent behavior in flocks. IEEE Trans. Autom. Control, 52, 852-862.

De Lellis, P., Di Bernardo, M. \& Garofalo, F. (2008) Synchronization of complex networks through local adaptive coupling. Chaos, 18, 037110.

De Lellis, P., di Bernardo, M., Gorochowski, T. E. \& Russo, G. (2010) Synchronization and control of complex networks via contraction, adaptation and evolution. IEEE Circuits Syst. Mag., 10, 64-82.

Donetti, L., Hurtado, P. I. \& NOz, M. A. M. (2005) Entangled networks, synchronization, and optimal network topology. Phys. Rev. Lett., 95, 188701.

Ermentrout, B. (1991) An adaptive model for synchrony in the firefly pteroptyx malaccae. J. Math. Biol., 29, 571-585.

Faria, J. J., Dyer, J. R., Clément, R. O., Couzin, I. D., Holt, N., Ward, A. J. W., Waters, D. \& Krause, J. (2010) A novel method for investigating the collective behaviour of fish: introducing 'Robofish'. Behav. Ecol. Sociobiol., 64, 1211-1218.

Frank, T. D. (2010) Active systems with nambu dynamics: with applications to rod wielding for haptic length perception and self-propagating systems on two-spheres. Eur. Phys. J. B, 74, 195-203.

Gorochowski, T. E., di Bernardo, M. \& Grierson, C. S. (2010) Evolving enhanced topologies for the synchronization of dynamical complex networks. Phys. Rev. E, 81, 056212.

HaN, J., LI, M. \& Guo, L. (2006) Soft control on collective behavior of a group of autonomous agents by a shill agent. J. Syst. Sci. Complex., 19, 54-62.

Hirsch, M. W., Smale, S. \& Devaney, R. L. (2004) Differential Equations, Dynamical Systems, and an Introduction to Chaos. San Diego: Academic Press.

Hongler, M.-O. \& Ryter, D. (1978) Hard mode stationary states generated by fluctuations. Zeitschrift für Physik $B, 31,333-337$.

Hramov, A. E., Khramova, A. E., Koronovskit, A. A. \& Boccaletti, S. (2008) Synchronization in networks of slightly nonidentical elements. Int. J. Bifurcation Chaos, 3, 845-850.

Hsien, M.-Y. A., Loizou, S. \& Kumar, V. (2007) Stabilization of multiple robots on stable orbits via local sensing. Proceedings of IEEE International Conference on Robotics and Automation (ICRA'07). New York: IEEE.

Liu, H., Chen, J., Lu, J-A. \& CAO, M. (2010) Generalized synchronization in complex dynamical networks via adaptive couplings. Physica A, 389, 1759-1770.

Liu, T., Hill, D. J. \& ZhaO, J. (2009) Synchronization of dynamical networks by network control. Proceedings of IEEE Conference on Decision and Control. New York: IEEE.

Mathews, P. M. \& Lakshmanan, M. (1974) On a unique nonlinear oscillator. Q. Appl. Math., 32, 215-218.

Motter, A., Zhou, C. \& Kurths, J. (2005) Enhancing complex-network synchronization. Europhys. Lett., 69, 334-340. 
Nambu, Y. (1973) Generalized hamiltonian dynamics. Phys. Rev. D, 7, 2405-2412.

Pecora, L. M. \& Carroll, T. L. (1998) Master stability functions for synchronized coupled systems. Phys. Rev. Lett., 80, 2109-2112.

Righetti, L., Buchli, J. \& IJsPeERT, A. J. (2006) Dynamic hebbian learning in adaptive frequency oscillators. Physica D, 216, 269-281.

Rodriguez, J. (2011) Networks of self-adaptive dynamical systems. Ph.D. thesis, Ecole Polytechnique Fédérale de Lausanne.

Rodriguez, J. \& Hongler, M.-O. (2009a) Networks of limit cycle oscillators with parametric learning capability. Recent Advances in Nonlinear Dynamics and Synchronization: Theory and Applications (K. Kyamakya, W. A. Halang, H. Unger, J. C. Chedjou, N. F. Rulkov \& Z. Li eds). Berlin: Springer, pp. 17-48.

Rodriguez, J. \& Hongler, M.-O. (2009b) Networks of mixed canonical-dissipative systems and dynamic hebbian learning. Int. J. Comput. Intell. Syst., 2, 140-146.

Rodriguez, J. \& Hongler, M.-O. (2010) Parametric resonance in time-dependent networks of hopf oscillators. Proceedings of European Conference on Complex Systems (ECCS'10). http://www.eccs2010.eu/publications.

Schweitzer, F. (2003) Brownian Agents and Active Particles. Berlin: Springer.

Schweitzer, F., Ebeling, W. \& Tilch, B. (2001) Statistical mechanics of canonical-dissipative systems and applications to swarm dynamics. Phys. Rev. E, 64, 021110.

TAylor, D., OtT, E. \& Restrepo, J. G. (2010) Spontanous synchronization of coupled oscillator systems with frequency adaptation. Phys. Rev. E, 81, 046214.

\section{Appendix A. Asymptotic stability of a compact set}

For a non-empty set $\mathcal{M} \subset \mathbb{R}^{p}$ and $x \in \mathbb{R}^{p}$, define the distance between $\mathcal{M}$ and $x$ by $\mathrm{N}(\mathcal{M}, x):=$ $\inf \{\|x-z\| \mid z \in \mathcal{M}\}\left(\|\cdot\|\right.$ the euclidean norm). For $t \in \mathbb{R}_{\geqslant 0}$, let $\varphi_{t}(x)$ be the flow of a dynamical system given by the ordinary differential equation $\dot{y}=\mathrm{L}(y)$ (i.e. $\varphi_{t}(x)=y(t)$ such that $\dot{y}(t)=\mathrm{L}(y(t))$ and $\left.\varphi_{0}(x)=y(0)=x\right)$.

Definition A.1 A set $\mathcal{M}$ is asymptotically stable if it is stable and it is attractor, that is

(1) Stable - if every neighbourhood $\mathcal{U}$ of $\mathcal{M}$ contains a set $\mathcal{V}$ that is a neighbourhood of $\mathcal{M}$ and $\mathcal{V}$ is positively invariant (i.e. $\varphi_{t}(x) \in \mathcal{V} \forall x \in \mathcal{V}, \forall t \geqslant 0$ ).

(2) Attractor-if the set $\mathcal{A}_{\mathcal{M}}:=\left\{x \in \mathbb{R}^{p} \mid \lim _{t \rightarrow \infty} \mathrm{N}\left(\mathcal{M}, \varphi_{t}(x)\right)=0\right\}$ is a neighbourhood of $\mathcal{M}$.

The well-known asymptotic stability result that is applied is (see Bhatia \& Szegö, 1970, Chapter VIII, Theorem 1.6) the following theorem.

Theorem A.2 Let $\mathcal{M} \subset \mathbb{R}^{p}$ be a non-empty compact set. If there exists a continuously differentiable real-valued function $\mathrm{F}(x)$ defined on a neighbourhood $\mathcal{U}$ of $\mathcal{M}$ such that

(1) $\mathrm{F}(x)=0$ if $x \in \mathcal{M}$ and $\mathrm{F}(x)>0$ if $x \notin \mathcal{M}$;

(2) $\langle\nabla \mathrm{F}(x) \mid \mathrm{L}(x)\rangle<0$ for $x \notin \mathcal{M}$;

then $\mathcal{M}$ is asymptotically stable. 


\section{Appendix B. Lemmas for symmetric and anti-symmetric matrices}

Lemma B.1 Let $L$ be an $N \times N$ symmetric matrix. Let $\mathrm{R}_{\mathrm{x}}$ and $\mathrm{R}_{\mathrm{y}}$ be two functions on $\mathbb{R}^{n}$ onto $\mathbb{R}^{N}$. Then

$$
\sum_{k=1}^{N} \sum_{j=1}^{N} l_{k, j}\left(\mathrm{R}_{\mathrm{x}_{j}}(X) \mathrm{R}_{\mathrm{y}_{k}}(X)-\mathrm{R}_{\mathrm{y}_{j}}(X) \mathrm{R}_{\mathrm{x}_{k}}(X)\right)=0 \quad \forall X .
$$

Proof. By direct calculation, we obtain

$$
\begin{aligned}
& \sum_{k=1}^{N} \sum_{j=1}^{N} l_{k, j}\left(\mathrm{R}_{\mathrm{x}_{j}}(X) \mathrm{R}_{\mathrm{y}_{k}}(X)-\mathrm{R}_{\mathrm{y}_{j}}(X) \mathrm{R}_{\mathbf{x}_{k}}(X)\right) \\
& \quad=\sum_{k=1}^{N}\left(\left(\sum_{j=1}^{N} l_{k, j} \mathrm{R}_{\mathbf{x}_{j}}(X)\right) \mathrm{R}_{\mathrm{y}_{k}}(X)-\left(\sum_{j=1}^{N} l_{k, j} \mathrm{R}_{\mathrm{y}_{j}}(X)\right) \mathrm{R}_{\mathrm{x}_{k}}(X)\right) \\
& =\left\langle L \mathrm{R}_{\mathbf{x}}(X) \mid \mathrm{R}_{\mathrm{y}}(X)\right\rangle-\left\langle L \mathrm{R}_{\mathbf{y}}(X) \mid \mathrm{R}_{\mathbf{x}}(X)\right\rangle \underbrace{=}_{L \text { is symmetric }}\left\langle L \mathrm{R}_{\mathbf{x}}(X) \mid \mathrm{R}_{\mathrm{y}}(X)\right\rangle-\left\langle\mathrm{R}_{\mathrm{y}}(X) \mid L \mathrm{R}_{\mathbf{x}}(X)\right\rangle=0 .
\end{aligned}
$$

Lemma B. 2 Let $T$ denote a $p \times p$ anti-symmetric matrix, $x, y \in \mathbb{R}^{p}$. Then

$$
\sum_{\substack{s, l=1 \\ s<l}}^{p} t_{l, s}\left(y_{l} x_{s}-y_{s} x_{l}\right)=\langle y \mid T x\rangle
$$

and therefore $\langle x \mid T x\rangle=0$.

Proof. Developing the left-hand side,

$$
\begin{aligned}
\sum_{\substack{s, l=1 \\
s<l}}^{p} t_{l, s}\left(y_{l} x_{s}-y_{s} x_{l}\right) & =\sum_{\substack{s, l=1 \\
s<l}}^{p} t_{l, s} y_{l} x_{s}+\sum_{\substack{s, l=1 \\
s<l}}^{p} \underbrace{-t_{l, s}}_{t_{s, l}} y_{s} x_{l}=\sum_{l=1}^{p-1} y_{l}\left(\sum_{s=l+1}^{p} t_{l, s} x_{s}\right)+\sum_{s=2}^{p} y_{s}\left(\sum_{l=1}^{s-1} t_{s, l} x_{l}\right) \\
& =\left\langle y \mid T^{\unlhd} x\right\rangle+\left\langle y \mid T_{\unrhd} x\right\rangle,
\end{aligned}
$$

where $T^{\unlhd}$ and $T_{\triangleright}$ are, respectively, upper and lower triangular matrices with the entries of $T$ implying that $T^{\unlhd}+T_{\unrhd}=T$, which concludes the proof.

\section{Appendix C. $\mathcal{M}$ is an $m$-dimensional compact submanifold of $\mathbb{R}^{p N}$}

Let $\mathrm{G}$ be defined as in (2.5) and denote $X \in \mathbb{R}^{p N}$ as $X:=\left(X_{1}, \ldots, X_{N}\right)$ with $X_{k}:=\left(x_{k, 1}, \ldots, x_{k, p}\right)$ and define $x_{k}:=\left(x_{1, k}, \ldots, x_{p, k}\right)$. Let $L$ be an $N \times N$ Laplacian matrix associated to a connected network ( $L:=D-A$ where entries of the adjacency matrix $A$ are symmetric and positive $a_{k, j}=a_{j, k} \geqslant 0$ for all $j, k$ and $D$ is a diagonal matrix with entries $d_{k, k}:=\sum_{j=1}^{N} a_{k, j}$ ). Finally, $\hat{L}$ is $L$ without its last line (i.e. an 
$(N-1) \times N$ matrix $)$. We have to show that

$$
\mathcal{M}:=\left\{X \in \mathbb{R}^{p N} \mid \mathrm{M}(X):=\left(\mathrm{G}\left(X_{1}\right), \hat{L} x_{1}, \ldots, \hat{L} x_{p}\right)=\mathbf{0} \in \mathbb{R}^{p N-m}\right\}
$$

is not empty, and that, for all $X^{*} \in \mathcal{M}$, there exists a neighbourhood $\mathcal{U}_{X^{*}} \subset \mathbb{R}^{p N}$ of $X^{*}$ such that $\mathfrak{D M}(X)$ has rank $p N-m$ for all $X \in \mathcal{U}_{X^{*}}$.

$[\mathcal{M} \neq \emptyset]$ The equations

$$
\hat{L} x_{1}=\cdots=\hat{L} x_{p}=\mathbf{0} \in \mathbb{R}^{N-1}
$$

are equivalent to

$$
x_{1,1}=\cdots=x_{N, 1}, x_{1,2}=\cdots=x_{N, 2}, \ldots, x_{1, p}=\cdots=x_{N, p} \Longleftrightarrow L x_{1}=\cdots=L x_{p}=\mathbf{0} \in \mathbb{R}^{N} .
$$

Obviously, Equations (C.2) imply Equations (C.1). It is also true the other way around since, for any $z \in \mathbb{R}^{p}$ such that $\hat{L} z=\mathbf{0}$, it follows that

$$
0=\sum_{k=1}^{N-1}\left(\sum_{j=1}^{N} l_{k, j} z_{j}\right)=\sum_{j=1}^{N} z_{j}\left(\sum_{k=1}^{N-1} l_{k, j}\right)=z_{N} \underbrace{\sum_{k=1}^{N-1} l_{k, N}}_{-l_{N, N}}+\sum_{j=1}^{N-1} z_{j}\left(\sum_{k=1}^{N-1} l_{k, j}\right)
$$

because $l_{s, s}=-\sum_{j \neq s}^{N} l_{s, j}$ and $l_{k, j}=l_{j, k}\left(l_{k}, j\right.$ entries of $\left.L\right)$. Since, for $j=1, \ldots, N-1, \sum_{k=1}^{N-1} l_{k, j}=l_{j, j}+$ $\sum_{k \neq j}^{N-1} l_{k, j}=-\sum_{j \neq s}^{N} l_{j, s}+\sum_{k \neq j}^{N-1} \underbrace{l_{k, j}}_{j, k}=-l_{j, N}$, then

$$
0=-l_{N, N} z_{N}+\sum_{j=1}^{N-1} z_{j}\left(-l_{j, N}\right)=-\sum_{j=1}^{N} l_{N, j} z_{j}
$$

and therefore $\hat{L} z=\mathbf{0}$ implies $L z=\mathbf{0}$. Hence, an element $X$ in $\mathcal{M}$ must satisfy $X_{k}=X_{c}$ for $k=1, \ldots, N$ and $\mathrm{G}\left(X_{c}\right)=\mathbf{0}$. Since by hypothesis $\mathcal{L}:=\left\{X \in \mathbb{R}^{p} \mid \mathrm{G}(X)=\mathbf{0} \in \mathbb{R}^{p-m}\right\}$ is a submanifold, it is not empty and therefore $\mathcal{M}$ is not empty as well.

$[\mathfrak{D M}(X)$ has rank $p N-m]$ For $X^{*}=\left(X_{1}^{*}, \ldots, X_{1}^{*}\right) \in \mathcal{M}$, consider a neighbourhood $\mathcal{U}_{X_{1}^{*}} \subset \mathbb{R}^{p}$ of $X_{1}^{*} \in \mathbb{R}^{p}$ such that $\mathfrak{D G}\left(X_{1}\right)$ has rank $p-m$ for all $X_{1} \in \mathcal{U}_{X_{1}^{*}}$ (such a neighbourhood exists since $\mathcal{L}$ is a submanifold of $\left.\mathbb{R}^{p}\right)$. Define $\mathcal{U}_{X^{*}}:=\mathcal{U}_{X_{1}^{*}} \times \cdots \times \mathcal{U}_{X_{1}^{*}}\left(p\right.$ times the Cartesian product of $\left.\mathcal{U}_{X_{1}^{*}}\right)$. Computing the derivative of $\mathrm{M}(X)$ and evaluating it on $X \in \mathcal{U}_{X^{*}}$ gives

$$
\mathfrak{D M}(X)=\left(\begin{array}{c}
\mathfrak{D G}\left(X_{1}\right) \mathbf{0} \\
Q
\end{array}\right)
$$

where $\mathfrak{D G}\left(X_{1}\right)$ is the $(p-m) \times p$ Jacobian of $\mathbf{G}, \mathbf{0}$ is a $(p-m) \times(N-1) p$ matrix with all entries 0 and $Q$ is a matrix with $p \times N$ blocks, each of size $N-1 \times p$ (i.e. $Q$ has $p(N-1)$ rows and $N p$ columns). Bloc $l, s$ has the sth column of matrix $\hat{L}$ in its $l$ th column and the rest of the entries are zero. We have to verify that the $N p-m$ lines of this Jacobian are linearly independent. Let $\beta_{j}(j=1, \ldots, p-m)$ and $\alpha_{j, k}$ 
$(j=1, \ldots, p, k=1, \ldots, N-1)$ be real numbers and we verify that they are all zeros if and only if

$$
\begin{aligned}
\sum_{j=1}^{N-1} l_{j, 1} \alpha_{s, j}+\sum_{j=1}^{p-m} \beta_{j} \frac{\partial \mathbf{G}_{j}}{\partial x_{s}}\left(X_{1}\right) & =0 \quad s=1, \ldots, p, \\
\sum_{j=1}^{N-1} l_{j, r} \alpha_{k, j} & =0 \quad k=1, \ldots, p, \quad r=2, \ldots, N .
\end{aligned}
$$

Since $L$ is symmetric $\left(l_{k, j}=l_{j, k}\right)$, Equations (C.3b) are equivalent to $\bar{L} \alpha_{k}=\mathbf{0} \in \mathbb{R}^{N-1}$ for $k=1, \ldots, p$, where $\bar{L}$ is $L$ without its first line and without its last column and $\alpha_{k}:=\left(\alpha_{k, 1}, \ldots, \alpha_{k, N-1}\right)$. Since the rank of $L$ is $N-1$ (Laplacian matrix associated to a connected network), the minor of $\bar{L}$ is non-zero, and therefore $\bar{L} \alpha_{k}=\mathbf{0}$ if and only if $\alpha_{k}=\mathbf{0}$ for $k=1, \ldots, p$. By hypothesis $X_{1} \in \mathcal{U}_{X_{1}^{*}}$ and $\mathfrak{D G}\left(X_{1}\right)$ has rank $p-m$, therefore $\beta_{j}=0 j=1, \ldots, p-m$. Hence, for $X \in \mathcal{U}_{X^{1}}, \mathfrak{D M}(X)$ has rank $p N-m$ and, thus, $\mathcal{M}$ has a dimension of $m$.

\section{Appendix D. Equality between kernels}

We have to see that, for all $X^{*} \in \mathcal{M}(\mathrm{cf} .(2.6))$, $\mathbb{k e r}\left(\mathfrak{D}^{2} \mathrm{~F}\left(X^{*}\right)\right)=\mathbb{k e r}\left(\mathfrak{D M}\left(X^{*}\right)\right)$ with

$$
\mathrm{F}(X):=\mathrm{K}(X)+\mathrm{V}(X)
$$

and where we define $\mathrm{K}(X):=\sum_{k=1}^{N}\left(1 / \mathrm{c}_{k}\right) \mathrm{E}_{k}\left(X_{k}\right)$ with $\mathrm{E}_{k}\left(X_{k}\right):=\frac{1}{2} \sum_{j \in I_{k}} \mathrm{G}_{j}\left(X_{k}\right)^{2}$. According to our definition of $\mathcal{M}$, any $X^{*} \in \mathcal{M}$ has the following property: $X_{1} \in \mathcal{L}$ (and therefore in any $\mathcal{L}_{k}$ ) and $X_{k}=X_{c}$ for all $k$.

Let $X^{*} \in \mathcal{M}$; then $\mathfrak{D}^{2} \mathrm{~F}\left(X^{*}\right)$ is positive semi-definite (since $X^{*}$ is a minimum) and symmetric. Therefore $\operatorname{ker}\left(\mathfrak{D}^{2} \mathrm{~F}\left(X^{*}\right)\right):=\left\{X \in \mathbb{R}^{p N} \mid\left\langle X \mid \mathfrak{D}^{2} \mathrm{~F}\left(X^{*}\right) X\right\rangle=0\right\}$ and $\left\langle X \mid \mathfrak{D}^{2} \mathrm{~F}\left(X^{*}\right) X\right\rangle=\left\langle X \mid \mathfrak{D}^{2} \mathrm{~K}\left(X^{*}\right) X\right\rangle+$ $\left\langle X \mid \mathfrak{D}^{2} \mathrm{~V}\left(X^{*}\right) X\right\rangle$, with both terms being positive semi-definite (since $X^{*}$ is a minimum for both terms). Hence

$$
X \in \mathbb{k} \operatorname{er}\left(\mathfrak{D}^{2} \mathrm{~F}\left(X^{*}\right)\right) \Longleftrightarrow\left\langle X \mid \mathfrak{D}^{2} \mathrm{~K}\left(X^{*}\right) X\right\rangle=0 \quad \text { and } \quad\left\langle X \mid \mathfrak{D}^{2} \mathrm{~V}\left(X^{*}\right) X\right\rangle=0
$$

where $\mathfrak{D}^{2} \mathrm{~K}(X)$ is an $N \times N$ block matrix with blocks of dimension $p \times p$ (i.e. $p N \times p N$ square matrix). The $k$ th diagonal block is the $p \times p$ symmetric matrix $\frac{1}{c_{k}} \mathfrak{D}^{2} E_{k}\left(X_{k}\right)$ and reads as

$$
\frac{1}{\mathbf{c}_{k}} \sum_{j \in I_{k}}\left(\frac{\partial \mathbf{G}_{j}}{\partial x_{k, s}}\left(X_{k}\right) \frac{\partial \mathbf{G}_{j}}{\partial x_{k, r}}\left(X_{k}\right)+\mathbf{G}_{j}\left(X_{k}\right) \frac{\partial^{2} \mathbf{G}_{j}}{\partial x_{k, s} \partial x_{r, k}}\left(X_{k}\right)\right) \quad r, s=1, \ldots, p,
$$

while all other entries are 0 . Evaluating $\mathfrak{D}^{2} \mathrm{~K}(X)$ at $X^{*} \in \mathcal{M}$ gives

$$
\frac{1}{\mathrm{c}_{k}} \sum_{j \in I_{k}}\left(\frac{\partial \mathrm{G}_{j}}{\partial x_{k, s}}\left(X_{k}^{*}\right) \frac{\partial \mathbf{G}_{j}}{\partial x_{k, r}}\left(X_{k}^{*}\right)\right) \text {. }
$$

We drop the $k$ index in the sum since it is no longer relevant (i.e. $X_{k}^{*}=X_{c}^{*}$ for all $k$ ) and use the notation $\partial \mathrm{G}_{j} / \partial x_{s}^{*}:=\left(\partial \mathrm{G}_{j} / \partial x_{k, s}\right)\left(X_{c}^{*}\right)$, so that the entries of the $p \times p$ symmetric matrix $\mathfrak{D}^{2} \mathbf{E}_{k}\left(X_{c}^{*}\right)$ are $\sum_{j \in I_{k}}\left(\partial \mathbf{G}_{j} / \partial x_{r}^{*}\right)\left(\partial \mathbf{G}_{j} / \partial x_{s}^{*}\right)$. Since $\mathfrak{D}^{2} \mathbf{E}_{k}\left(X_{c}^{*}\right)$ is positive semi-definite (since $X_{c}^{*}$ is a minimum) for all $k$, 
we have

$$
\left\langle X \mid \mathfrak{D}^{2} \mathrm{~K}\left(X^{*}\right) X\right\rangle=0 \Longleftrightarrow\left\langle X_{k} \mid \frac{1}{\mathrm{c}_{k}} \mathfrak{D}^{2} \mathrm{E}_{k}\left(X_{c}^{*}\right) X_{k}\right\rangle=0 \quad \text { for all } k
$$

As we obtain

$$
\begin{aligned}
\left\langle X_{k} \mid \frac{1}{\mathbf{c}_{k}} \mathfrak{D}^{2} \mathbf{E}_{k}\left(X_{c}^{*}\right) X_{k}\right\rangle & =\frac{1}{\mathbf{c}_{k}} \sum_{l \in I_{k}}\left(\sum_{r=1}^{p} \sum_{s=1}^{p} \frac{\partial \mathbf{G}_{l}}{\partial x_{r}^{*}} \frac{\partial \mathbf{G}_{l}}{\partial x_{s}^{*}} x_{k, s} x_{k, r}\right) \\
& =\frac{1}{\mathbf{c}_{k}} \sum_{l \in I_{k}}\left(\sum_{j=1}^{p}\left(\frac{\partial \mathbf{G}_{l}}{\partial x_{j}^{*}} x_{k, j}\right)^{2}+2 \sum_{\substack{r, j=1 \\
r<j}}^{p} \frac{\partial \mathbf{G}_{l}}{\partial x_{r}^{*}} x_{k, r} \frac{\partial \mathbf{G}_{l}}{\partial x_{j}^{*}} x_{k, j}\right) \\
& =\frac{1}{\mathbf{c}_{k}} \sum_{l \in I_{k}}\left(\sum_{j=1}^{p} \frac{\partial \mathbf{G}_{l}}{\partial x_{j}^{*}} x_{k, j}\right)^{2},
\end{aligned}
$$

it follows that

$$
\left\langle X \mid \mathfrak{D}^{2} \mathrm{~K}\left(X^{*}\right) X\right\rangle=0 \Longleftrightarrow\left\langle\nabla \mathrm{G}_{l}\left(X_{c}^{*}\right) \mid X_{k}\right\rangle=0 \quad l \in I_{k} \quad \forall k \Longleftrightarrow X_{k} \in \mathbb{k} \operatorname{er}\left(\mathfrak{D G}\left(X^{*}\right)\right) \forall k .
$$

By hypothesis (2.4), we have that $\left\langle X \mid \mathfrak{D}^{2} \mathrm{~V}\left(X^{*}\right) X\right\rangle=0 \Longleftrightarrow X_{k}=X_{c}$ for all $k$, and so

$$
X \in \mathbb{k} \operatorname{er}\left(\mathfrak{D}^{2} \mathrm{~F}\left(X^{*}\right)\right) \Longleftrightarrow X_{k}=X_{c} \forall k \text { and } X_{c} \in \mathbb{k} \operatorname{er}\left(\mathfrak{D G}\left(X^{*}\right)\right) \Longleftrightarrow X \in \mathbb{k} \operatorname{er}\left(\mathfrak{D M}\left(X^{*}\right)\right) \text {. }
$$

\section{Appendix E. Existence of set $\mathcal{U}$}

To prove Corollary E.2, we first need to consider the following Lemma. In words, it states that, for the gradient of a real-valued function $\mathrm{F}$ not to vanish in a neighbourhood of a manifold $\mathcal{M}$, the kernel of F's Hessian must be the same as the kernel of $\mathcal{M}$.

Lemma E. 1 Let $\mathcal{M}$ be a submanifold given by $\mathrm{M}: \mathbb{R}^{m} \rightarrow \mathbb{R}^{m-k}$ (i.e. $\mathcal{M}$ has a dimension of $k$ ). Here $\mathrm{F}$ is a real-valued function on $\mathbb{R}^{m}$ such that $\mathrm{F}(x) \geqslant 0$ for all $x$ and $\mathrm{F}(x)=0$ if and only if $x \in \mathcal{M}$. Suppose that there exists $x^{*} \in \mathcal{M}$ such that $\mathbb{k} \operatorname{er}\left(\mathfrak{D}^{2} \mathrm{~F}\left(x^{*}\right)\right)=\mathbb{k} \operatorname{er}\left(\mathfrak{D M}\left(x^{*}\right)\right)$. Then there exists an open set $\mathcal{U}_{x^{*}} \ni x^{*}$ such that $\nabla \mathrm{F}(x) \neq \mathbf{0}$ for all $x \in \mathcal{U}_{x^{*}} \backslash \mathcal{M}$.

Proof. By hypothesis there exists $x^{*} \in \mathcal{M}$ such that $\mathbb{k e r}\left(\mathfrak{D}^{2} \mathrm{~F}\left(x^{*}\right)\right)=\mathbb{k} \operatorname{er}\left(\mathfrak{D M}\left(x^{*}\right)\right)$. Without loss of generality, we can chose a basis of $\mathbb{R}^{m}$ such that the first $k$ basis vectors span the kernel of $\mathfrak{D M}\left(x^{*}\right)$, and we use the following notation: $x=(\bar{x}, \hat{x})$. In this basis, $(\partial \mathrm{M} / \partial \hat{x})\left(x^{*}\right)^{-1}$ exists and, since $\mathrm{M}\left(x^{*}\right)=0$, the implicit function theorem guarantees the existence of $r_{\mathrm{M}}, R_{\mathrm{M}}>0$ and a unique continuous map $\mathrm{I}_{\mathrm{M}}: \mathcal{B}\left(\bar{x}^{*}, r_{\mathrm{M}}\right) \longrightarrow \mathcal{B}\left(\hat{x}^{*}, R_{\mathrm{M}}\right)$ such that

$$
\mathrm{M}(\bar{x}, \hat{x})=0 \Longleftrightarrow \mathrm{I}_{\mathrm{M}}(\bar{x})=\hat{x} \quad \forall(\bar{x}, \hat{x}) \in \mathcal{B}\left(\bar{x}^{*}, r_{\mathrm{M}}\right) \times \mathcal{B}\left(\hat{x}^{*}, R_{\mathrm{M}}\right),
$$

where $\mathcal{B}\left(x^{*}, r\right)$ is the open ball centred at a point $x^{*}$ and of radius $r>0$. Define

$$
\begin{aligned}
\mathrm{S}: \mathbb{R}^{k} \times \mathbb{R}^{m-k} & \longrightarrow \mathbb{R}^{m-k} \\
(\bar{x}, \hat{x}) \longmapsto & \pi(\nabla \mathrm{F}(x)),
\end{aligned}
$$


with projection $\pi\left(x_{1}, \ldots, x_{m}\right)=\left(x_{k+1}, \ldots, x_{m}\right)$. By hypothesis $\mathbb{k e r}\left(\mathfrak{D}^{2} \mathrm{~F}\left(x^{*}\right)\right)=\mathbb{k} \operatorname{er}\left(\mathfrak{D M}\left(x^{*}\right)\right)$ and so $(\partial \mathrm{S} / \partial \hat{x})\left(x^{*}\right)^{-1}$ exists. Since $\mathcal{M}$ is the minimum of $\mathrm{F}$, we have $\mathcal{M} \subseteq \nabla \mathrm{F}^{-1}(0) \subseteq \mathrm{S}^{-1}(0)$ and so $\mathrm{S}\left(x^{*}\right)=0$. Therefore, applying the implicit function theorem, there exists $r_{\mathrm{S}}, R_{\mathrm{S}}>0$ and a unique continuous map $\mathrm{I}_{\mathrm{S}}: \mathcal{B}\left(\bar{x}^{*}, r_{\mathrm{S}}\right) \longrightarrow \mathcal{B}\left(\hat{x}^{*}, R_{\mathrm{S}}\right)$ such that

$$
\mathrm{S}(\bar{x}, \hat{x})=0 \Longleftrightarrow \mathrm{I}_{\mathrm{S}}(\bar{x})=\hat{x} \quad \forall(\bar{x}, \hat{x}) \in \mathcal{B}\left(\bar{x}^{*}, r_{\mathrm{S}}\right) \times \mathcal{B}\left(\hat{x}^{*}, R_{\mathrm{S}}\right)
$$

Define $\mathcal{B}:=\mathcal{B}\left(\bar{x}^{*}, r\right) \times \mathcal{B}\left(\hat{x}^{*}, R\right)$ with $r:=\min \left\{r_{\mathrm{M}}, r_{\mathrm{S}}\right\}$ and $R:=\min \left\{R_{\mathrm{M}}, R_{\mathrm{S}}\right\}$ and let

$$
\operatorname{Graph}\left(\mathrm{I}_{\mathrm{M}}\right):=\left\{\left(\bar{x}, \mathrm{I}_{\mathrm{M}}(\bar{x})\right) \in \mathbb{R}^{m} \mid \bar{x} \in \mathcal{B}\left(\bar{x}^{*}, r\right)\right\}
$$

and

$$
\operatorname{Graph}\left(\mathrm{I}_{\mathrm{S}}\right):=\left\{\left(\bar{x}, \mathrm{I}_{\mathrm{S}}(\bar{x})\right) \in \mathbb{R}^{m} \mid \bar{x} \in \mathcal{B}\left(\bar{x}^{*}, r\right)\right\} .
$$

By the implicit function theorem, $\operatorname{Graph}\left(\mathrm{I}_{M}\right):=\mathcal{M} \cap \mathcal{B}$ and $\mathbb{G r a p h}\left(\mathrm{I}_{S}\right):=\mathrm{S}^{-1}(0) \cap \mathcal{B}$. Since $\mathcal{M} \subseteq$ $\nabla \mathrm{F}^{-1}(0) \subseteq \mathrm{S}^{-1}(0)$, it follows that $\mathcal{M} \cap \mathcal{B} \subseteq \nabla \mathrm{F}^{-1}(0) \cap \mathcal{B} \subseteq \mathrm{S}^{-1}(0) \cap \mathcal{B}$ and so

$$
\mathbb{G r a p h}\left(\mathrm{I}_{\mathrm{M}}\right) \subseteq \mathbb{G} \operatorname{raph}\left(\mathrm{I}_{\mathrm{S}}\right)
$$

In fact, these two sets are equal. To see this, suppose that there exists $z=(\bar{z}, \hat{z}) \in \mathcal{B}$ such that $z \in$ $\mathbb{G r a p h}\left(\mathrm{I}_{S}\right)$ but $z \notin \mathbb{G r a p h}\left(\mathrm{I}_{\mathrm{M}}\right)$. Since $z \in \mathbb{G r a p h}\left(\mathrm{I}_{S}\right)$, then $\mathrm{I}_{\mathrm{S}}(\bar{z})=\hat{z}$ and since $z \notin \mathbb{G r a p h}\left(\mathrm{I}_{\mathrm{M}}\right)$, it follows that $I_{M}(\bar{z}) \neq \hat{z}$. However, since (E.4), it follows that $\left(\bar{z}, I_{M}(\bar{z})\right) \in \mathbb{G r a p h}\left(I_{h}\right)$, which implies (because $I_{S}$ is a map) that

$$
\mathrm{I}_{\mathrm{S}}(\bar{z})=\mathrm{I}_{\mathrm{M}}(\bar{z}) \neq \hat{z}
$$

which is a contradiction. Therefore $\operatorname{Graph}\left(\mathrm{I}_{\mathrm{M}}\right)=\mathbb{G r a p h}\left(\mathrm{I}_{\mathrm{S}}\right)$, so $\mathcal{M} \cap \mathcal{B}=\mathrm{S}^{-1}(0) \cap \mathcal{B}$ and hence $\mathcal{M} \cap \mathcal{B}=\nabla \mathrm{F}^{-1}(0) \cap \mathcal{B}$. Thus, there exists an open set $\mathcal{U}_{x^{*}} \ni x^{*}$ which is $\mathcal{B}$ such that, for all $x \in$ $\mathcal{U}_{x^{*}} \backslash \mathcal{M}, \nabla \mathrm{F}(x) \neq 0$.

Corollary E.2 With the same hypothesis as in Lemma (E.1) and supposing additionally that for all $x^{*} \in \mathcal{M}$, we have $\mathbb{k e r}\left(\mathfrak{D} \nabla \mathrm{F}\left(x^{*}\right)\right)=\mathbb{k e r}\left(\mathfrak{D M}\left(x^{*}\right)\right)$. Then, there exists an open set $\mathcal{U} \supset \mathcal{M}$ such that $\nabla \mathrm{F}(x) \neq \mathbf{0}$ for all $x \in \mathcal{U} \backslash \mathcal{M}$.

Proof. By Lemma (E.1), there exists an open $\operatorname{set} \mathcal{U}_{x^{*}} \ni x^{*} \operatorname{such}$ that $\nabla \mathrm{F}(x) \neq 0$ for all $x \in \mathcal{U}_{x^{*}} \backslash \mathcal{M}$. Then $\mathcal{U}$ is given by

$$
\mathcal{U}:=\bigcup_{x^{*} \in \mathcal{M}} \mathcal{U}_{x^{*}}
$$

\section{Appendix F. Proofs of Propositions 3.2, 3.4 and 3.6}

Proof of Proposition 3.2. The convergence towards $\mathcal{C}_{\lambda_{\mathrm{c}}}$ follows from Lyapunov's second method with Lyapunov function

$$
\mathrm{F}_{\lambda_{\mathrm{c}}}(X, \Lambda):=\mathrm{F}(X)+\frac{1}{2} \sum_{k=1}^{N} \frac{\left(\lambda_{k}-\lambda_{\mathrm{c}}\right)^{2}}{\mathrm{~s}_{k}} \geqslant 0
$$


where $\mathrm{F}(X)$ is defined in Lemma 3.1. By construction, we have $\mathcal{C}_{\lambda_{\mathrm{c}}}=\left\{(X, \Lambda) \in \mathbb{R}^{N p} \times \mathbb{R}^{N} \mid \mathrm{F}(X, \Lambda)=\right.$ $0\}$. Computing the time derivative,

$$
\begin{aligned}
\left\langle\nabla \mathrm{F}_{\lambda_{\mathrm{c}}}(X, \Lambda) \mid(\dot{X}, \dot{\Lambda})\right\rangle= & \sum_{k=1}^{N}\left\langle\frac{1}{\mathrm{c}_{k}} \nabla \mathrm{E}_{k}\left(X_{k}\right)+\frac{\partial \mathrm{V}}{\partial X_{k}}(X) \mid \dot{X}_{k}\right\rangle+\sum_{k=1}^{N} \frac{\left(\lambda_{k}-\lambda_{\mathrm{c}}\right)}{\mathrm{s}_{k}} \dot{\lambda}_{k} \\
= & \sum_{k=1}^{N}\left\langle\frac{1}{\mathrm{c}_{k}} \nabla \mathrm{E}_{k}\left(X_{k}\right)+\frac{\partial \mathrm{V}}{\partial X_{k}}(X) \mid \lambda_{k} \mathrm{~K}\left(X_{k}\right)-\nabla \mathrm{E}_{k}\left(X_{k}\right)-\mathrm{c}_{k} \frac{\partial \mathrm{V}}{\partial X_{k}}(X)\right\rangle \\
& -\sum_{k=1}^{N} \lambda_{k}\left\langle\frac{\partial \mathrm{V}}{\partial X_{k}}(X) \mid \mathrm{K}\left(X_{k}\right)\right\rangle+\lambda_{\mathrm{c}} \underbrace{N}_{k=1}\left\langle\frac{\partial \mathrm{V}}{\partial X_{k}}(X) \mid \mathrm{K}\left(X_{k}\right)\right\rangle \\
= & \sum_{k=1}^{N} \frac{1}{\mathrm{c}_{k}} \underbrace{\left\langle\nabla \mathrm{E}_{k}\left(X_{k}\right) \mid \lambda_{k} \mathrm{~K}\left(X_{k}\right)\right\rangle}_{=0}+\sum_{k=1}^{N}\left\langle\frac{\partial \mathrm{V}}{\partial X_{k}}(X) \mid \lambda_{k} \mathrm{~K}\left(X_{k}\right)\right\rangle \\
& \underbrace{-\sum_{k=1}^{N} \mathrm{c}_{k}\left\|\frac{1}{\mathrm{c}_{k}} \nabla \mathrm{E}_{k}\left(X_{k}\right)+\frac{\partial \mathrm{V}}{\partial X_{k}}(X)\right\|^{2}}_{\leqslant=0}-\sum_{k=1}^{N}\left\langle\frac{\partial \mathrm{V}}{\partial X_{k}}(X) \mid \lambda_{k} \mathrm{~K}\left(X_{k}\right)\right\rangle .
\end{aligned}
$$

Let $\mathcal{U}_{\lambda_{\mathrm{c}}}$ be a neighbourhood of $\lambda_{\mathrm{c}} \mathbf{1}$ included in the hyperplane

$$
\left\{\Lambda \in \mathbb{R}^{N} \mid \sum_{k=1}^{N} \frac{\lambda_{k}}{\mathbf{s}_{k}}=\lambda_{\mathrm{c}} \sum_{k=1}^{N} \frac{1}{\mathbf{s}_{k}}\right\} .
$$

Therefore, by taking the open set $\mathcal{U} \supset \mathcal{M}$ whose existence we have proved in Lemma 3.1, strict negativity of $\left\langle\nabla \mathrm{F}_{\lambda_{\mathrm{c}}}(X, \Lambda) \mid(\dot{X}, \dot{\Lambda})\right\rangle<0$ holds for all $(X, \Lambda) \in \mathcal{U} \times \mathcal{U}_{\lambda_{\mathrm{c}}} \backslash \mathcal{C}_{\lambda_{\mathrm{c}}}$. Hence, the compact set $\mathcal{C}_{\lambda_{\mathrm{c}}}$ is asymptotically stable (see Appendix A for details).

The hypothesis that $\sum_{k=1}^{N}\left\langle\left(\partial \mathrm{V} / \partial X_{k}\right)(X) \mid \mathrm{K}\left(X_{k}\right)\right\rangle=0$ leads to the existence of a constant of motion: $\mathrm{J}\left(\lambda_{1}, \ldots, \lambda_{N}\right):=\sum_{k=1}^{N}\left(\lambda_{k}(t) / \mathrm{s}_{k}\right)$. Indeed, for $\left\{\lambda_{k}(t)\right\}_{k=1}^{N}$ orbits of Equations (3.1), we have

$$
\frac{\mathrm{d}\left[\mathrm{J}\left(\lambda_{1}(t), \ldots, \lambda_{N}(t)\right)\right]}{\mathrm{d} t}=\sum_{k=1}^{N} \frac{\dot{\lambda}_{k}}{\mathrm{~s}_{k}}=-\sum_{k=1}^{N}\left\langle\frac{\partial \mathrm{V}}{\partial X_{k}}(X) \mid \mathrm{K}\left(X_{k}\right)\right\rangle=0
$$

Thus $\mathbf{C}=\sum_{k=1}^{N}\left(\lambda_{k}(t) / \mathbf{s}_{k}\right)$ for all $t$, and $\mathbf{C}=\sum_{k=1}^{N}\left(\lambda_{k}(0) / \mathbf{s}_{k}\right)$. Owing to the convergence, $\mathbf{C}=$ $\lambda_{\mathrm{c}} \sum_{k=1}^{N}\left(1 / \mathrm{s}_{k}\right)$ and therefore

$$
\lambda_{\mathrm{c}}:=\frac{\sum_{k=1}^{N}\left(\lambda_{k}(0) / \mathrm{s}_{k}\right)}{\sum_{k=1}^{N}\left(1 / \mathrm{s}_{k}\right)}
$$


Proof of Proposition 3.4. The convergence towards $\mathcal{C}_{\Lambda_{\mathrm{c}}}$ follows from Lyapunov's second method with Lyapunov function

$$
\mathrm{F}_{\Lambda_{\mathrm{c}}}(X, \Lambda):=\mathrm{F}(X)+\frac{1}{2} \sum_{\substack{s, l=1 \\ s<l}}^{p}\left(\sum_{k=1}^{N} \frac{\left(\lambda_{k, l, s}-\lambda_{c, l, s}\right)^{2}}{\mathrm{~s}_{k, l, s}}\right) \geqslant 0
$$

where $\mathrm{F}(X)$ is defined in Lemma 3.1 with here $\mathrm{E}_{k}\left(X_{k}\right)=\frac{1}{2} \mathrm{G}\left(X_{k}\right)^{2}$. The rest of the proof is similar to the one in Proposition 3.2. Full calculations are found in Rodriguez (2011).

Proof of Proposition 3.6. The convergence towards $\mathcal{C}_{\lambda_{\mathrm{c}} \times \Lambda_{\mathrm{c}}}$ follows from Lyapunov's second method with Lyapunov function:

$$
\mathrm{F}_{\lambda_{\mathrm{c}} \times \Lambda_{\mathrm{c}}}(X, \Lambda):=\mathrm{F}(X)+\frac{1}{2} \sum_{k=1}^{v} \frac{\left(\lambda_{k}-\lambda_{\mathrm{c}}\right)^{2}}{\mathbf{s}_{k}}+\frac{1}{2} \sum_{\substack{s, l=1 \\ s<l}}^{p}\left(\sum_{k=v+1}^{N} \frac{\left(\lambda_{k, l, s}-\lambda_{c}\right)^{2}}{\mathbf{s}_{k, l, s}}\right),
$$

where $\mathrm{F}(X)$ is defined as in Appendix D with $\mathrm{E}_{k}\left(X_{k}\right):=\frac{1}{2} \sum_{j \in I_{k}} \mathrm{G}_{j}\left(X_{k}\right)^{2}$ for $k=1, \ldots, v$ and $\mathrm{E}_{k}\left(X_{k}\right):=$ $\frac{1}{2} \mathrm{G}_{1}\left(X_{k}\right)^{2}$ for $k=v+1, \ldots, N$. The rest of the proof is similar to the one in Proposition 3.2. Full calculations are found in Rodriguez (2011). 